\title{
LHCb VErtex LOcator (VELO) Module Production and Performance
}

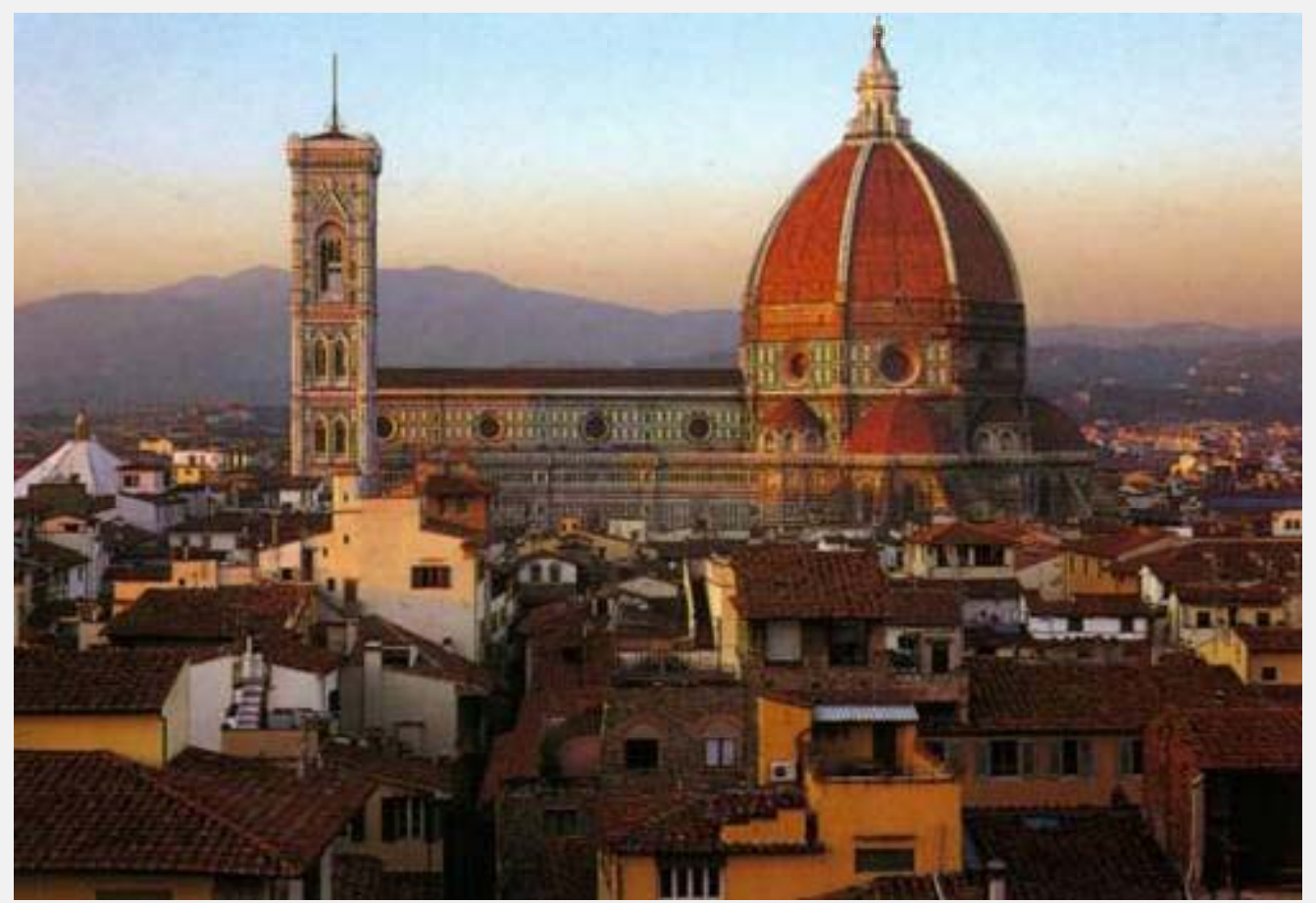

Anthony Affolder

University of Liverpool

for the LHCb VELO group

RD07 Conference

June 27, 2007 


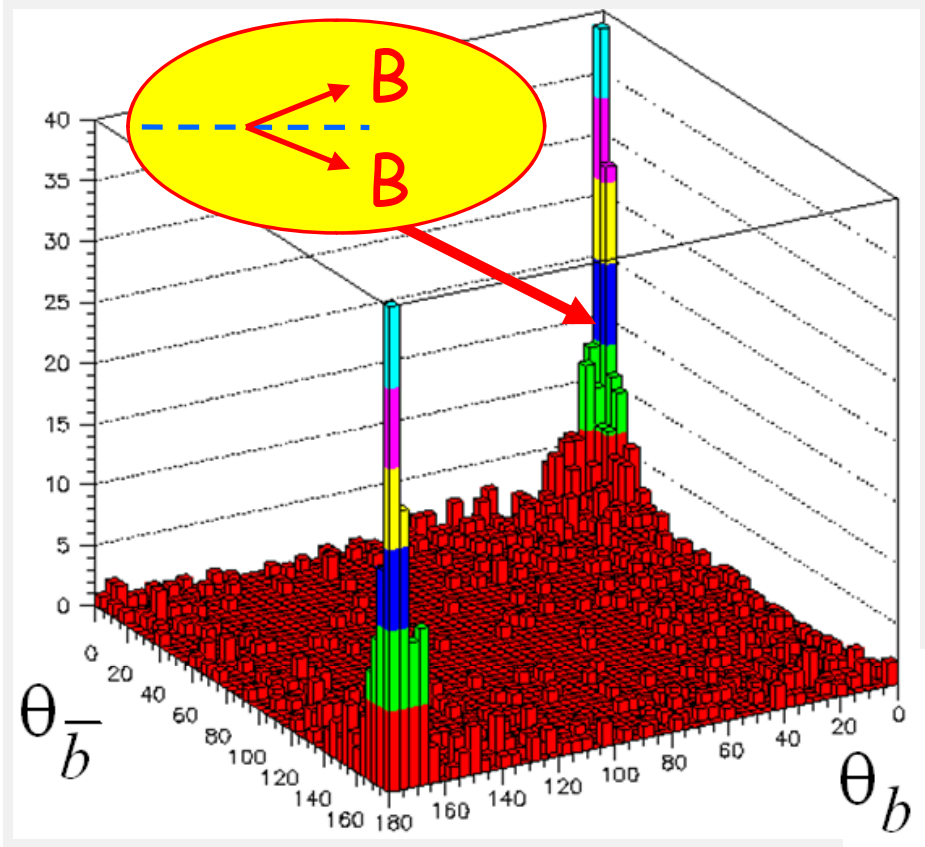

- At LHC, bottom production is peaked at high rapidity with both quarks in the same direction

- LHCb built as forward spectrometer to optimize for the study of bottom quark decays

- The VErtex LOcator (VELO) is the silicon microstrip vertexer at the collision point

This talk focuses on the VELO module production \& performance

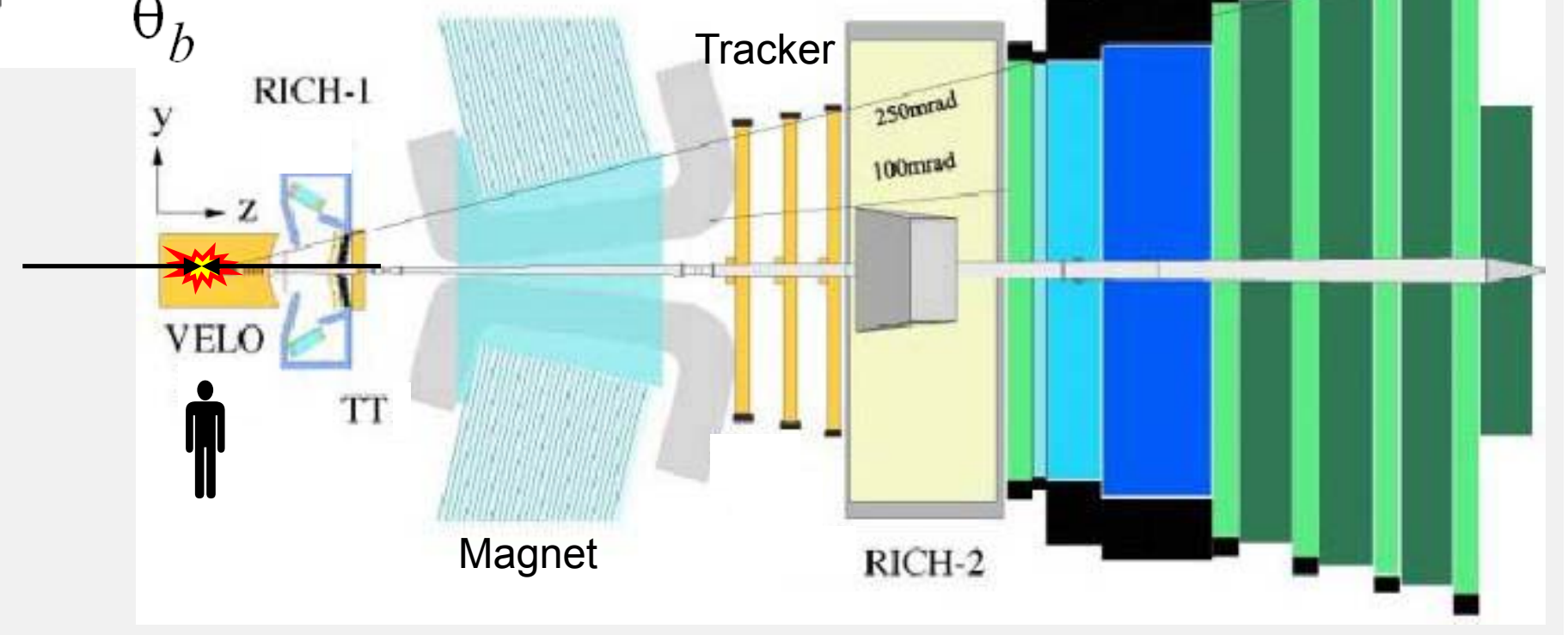




\section{LHCb
IHCLO Design Requirements}

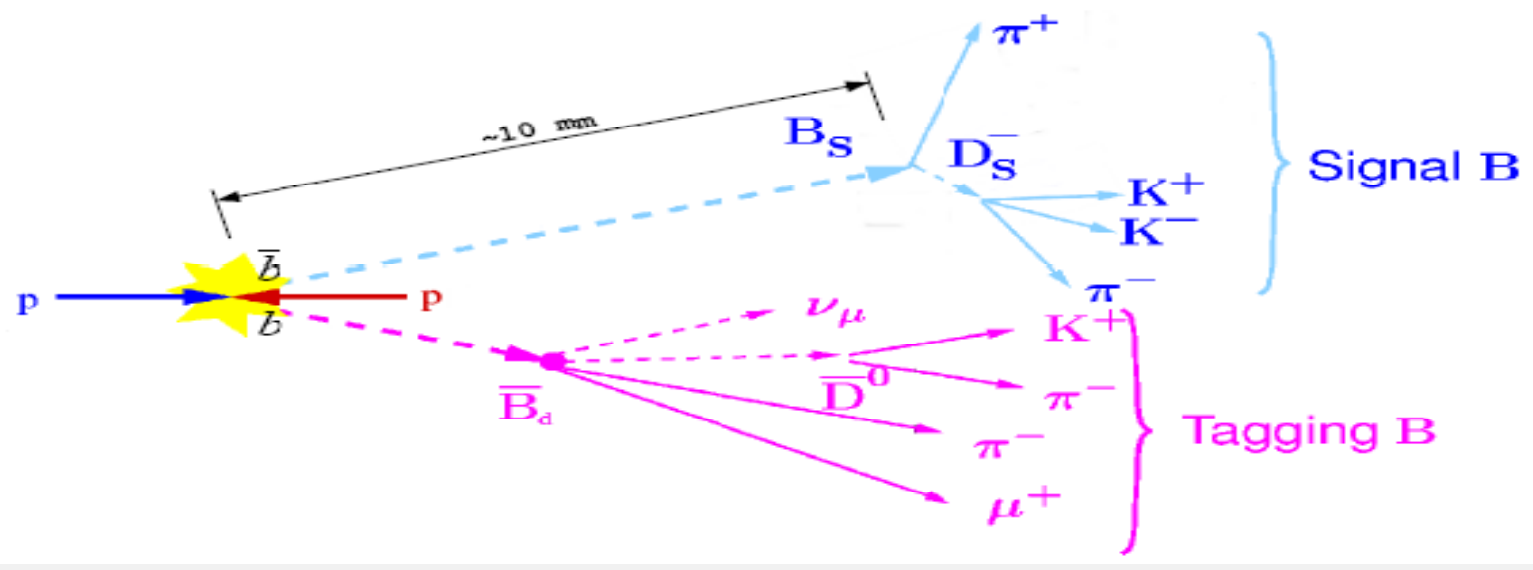

\section{Vertexing}

$>$ Need to separate (multiple) primary and secondary vertices $(<100 \mu \mathrm{m}$ resolution $\|$ beams)

- Close to LHC beam $(8 \mathrm{~mm}) \rightarrow$ Vacuum

- Extreme radiation levels $\sim 10^{14}$ neq $/ \mathrm{cm}^{2} /$ year @inner radius $\rightarrow$ n-strip silicon sensors

- Prevent reverse annealing $\left(<-5^{\circ} \mathrm{C}\right)$ $\rightarrow \mathrm{CO}_{2}$ cooling

Tracking

$>$ Impact parameter $\sim 40 \mu \mathrm{m}$ (40 fs time resolution) - Low mass $\sim 15 \% \mathrm{X}_{\mathrm{O}}$

\section{Trigger}

$>$ Fast computation of primary vertices and impact parameter

- R-phi sensor geometry

- Tight mechanical tolerances (Limit: $40 \mu \mathrm{m} \perp$ beams, $200 \mu \mathrm{m} \|$ beams)

\section{Beams}

$>$ Injection Clearance $\mathcal{O}(30 \mathrm{~mm})$ for each half

- Moving detectors

\section{Bespoke, severely constrained module design}


-All 42 installed modules on base

$>$ Delivery finished Feb. 2007

-Installation at pit late summer 2007

-Data taking starts spring 2008

- Consists of:

$>$ Carbon fibre clad TPG hybrid core

$>$ Laminated with flex hybrids

$>2$ sensors ( $R$ \& phi geometry)

$>$ Carbon-fibre paddle

$>$ Precision base

$>$ Kapton cables

$>$ Silver-plated ground straps

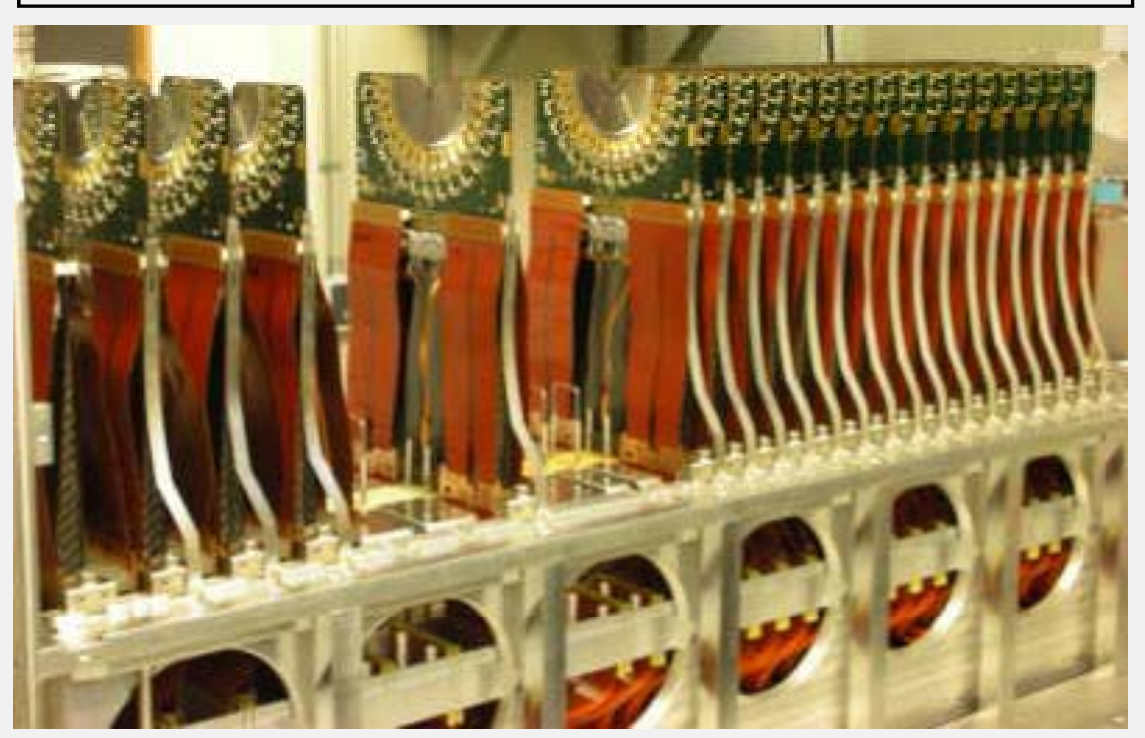

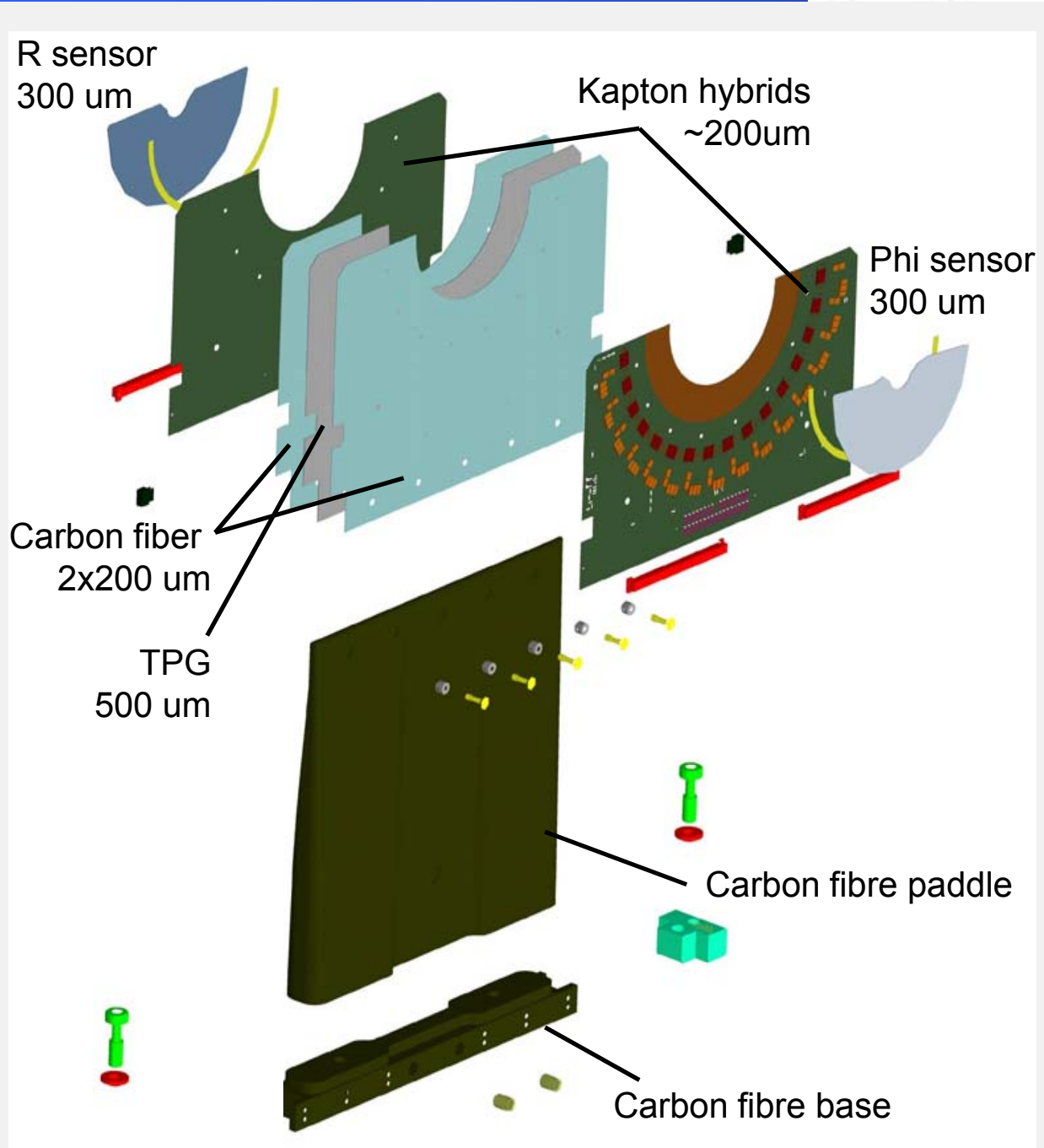

Invar feet 


\section{Sensors}

-n strip sensor technology (Micron)

$>\mathrm{n}$ bulk-46 modules (43 installed)

$>$ p bulk-2 modules ( 1 installed)

-Double metal for signal routing

-Closest active strip $8.2 \mathrm{~mm}$ from beam

- R sensors

$>4$ quadrants

$>$ Pitch from $40 \mu \mathrm{m}$ to $101 \mu \mathrm{m}$

-Phi sensor

$>$ Divided into inner/outer sensor

$>$ Pitch from $35 \mu \mathrm{m}$ to $96 \mu \mathrm{m}$

$>$ Stereo angle

$--20^{\circ}$ inner, $10^{\circ}$ outer

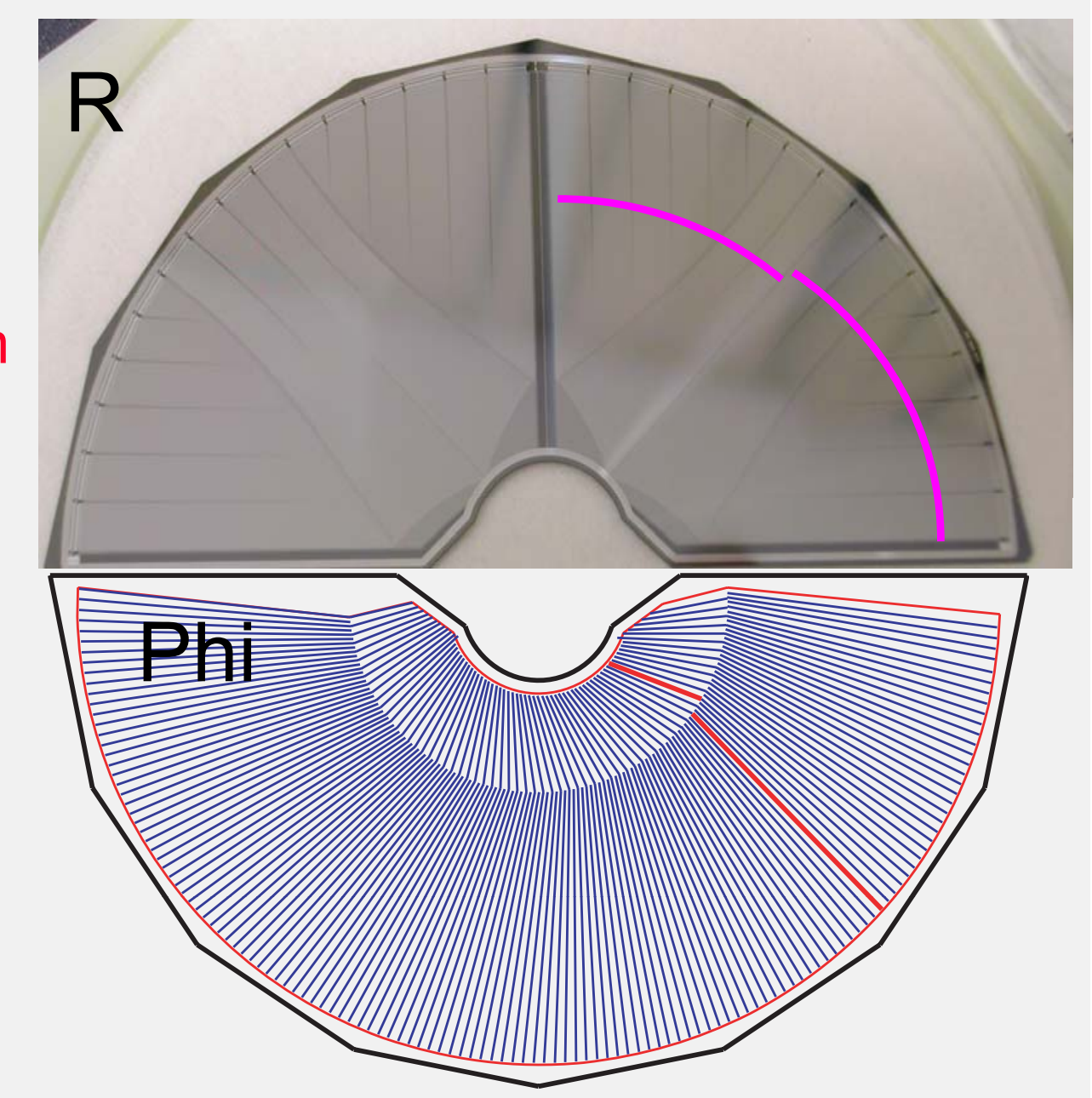

-0.3\% faulty strips in production sensors 


\section{LHCb Radiation Hardness}

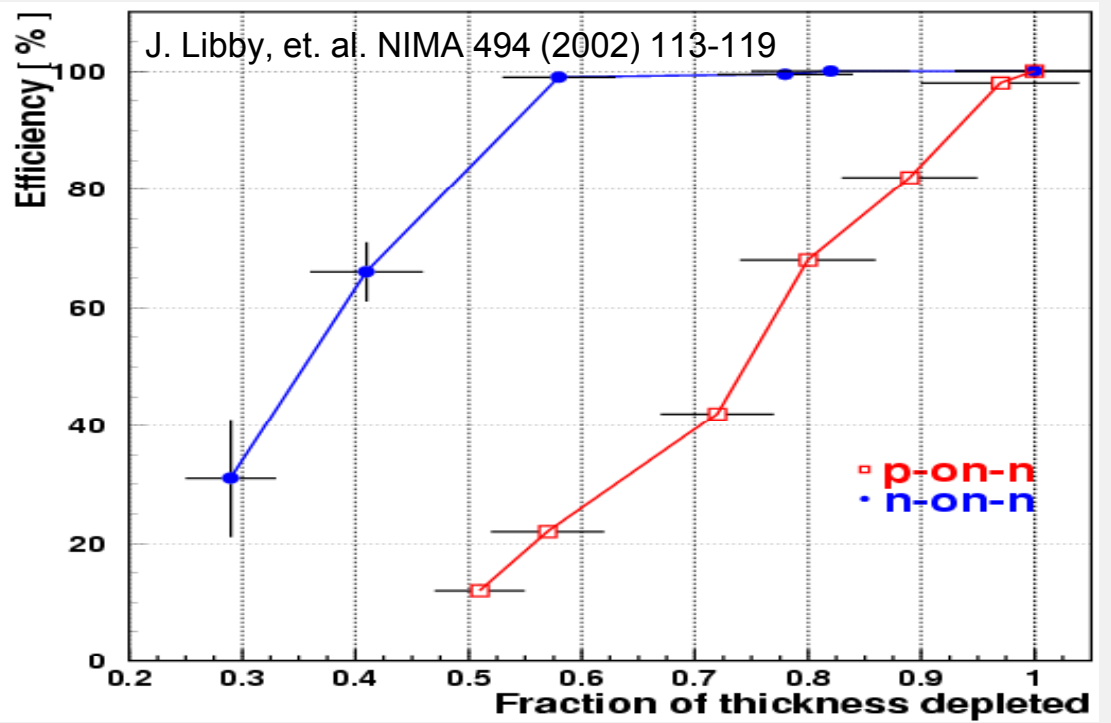

•n strip sensors inherently radiation hard

$>$ Collecting electrons increases trapping times/mean free path

$>$ With p-spray, isolated even when partially depleted

-After 3-4 years (6-8 $\left.\mathrm{fb}^{-1}\right)$, the inner region of the sensor cannot be fully depleted

\section{- Dose estimates}

- $1.3 * 10^{14} \mathrm{neq} / \mathrm{cm}^{2} /$ year at $\mathrm{R}=8 \mathrm{~mm}$

- 5 * $10^{12} \mathrm{neq} / \mathrm{cm}^{2} /$ year at $\mathrm{R}=42 \mathrm{~mm}$

-Running partially depleted, the estimated lifetime is $\sim 16 \mathrm{fb}^{-1}$
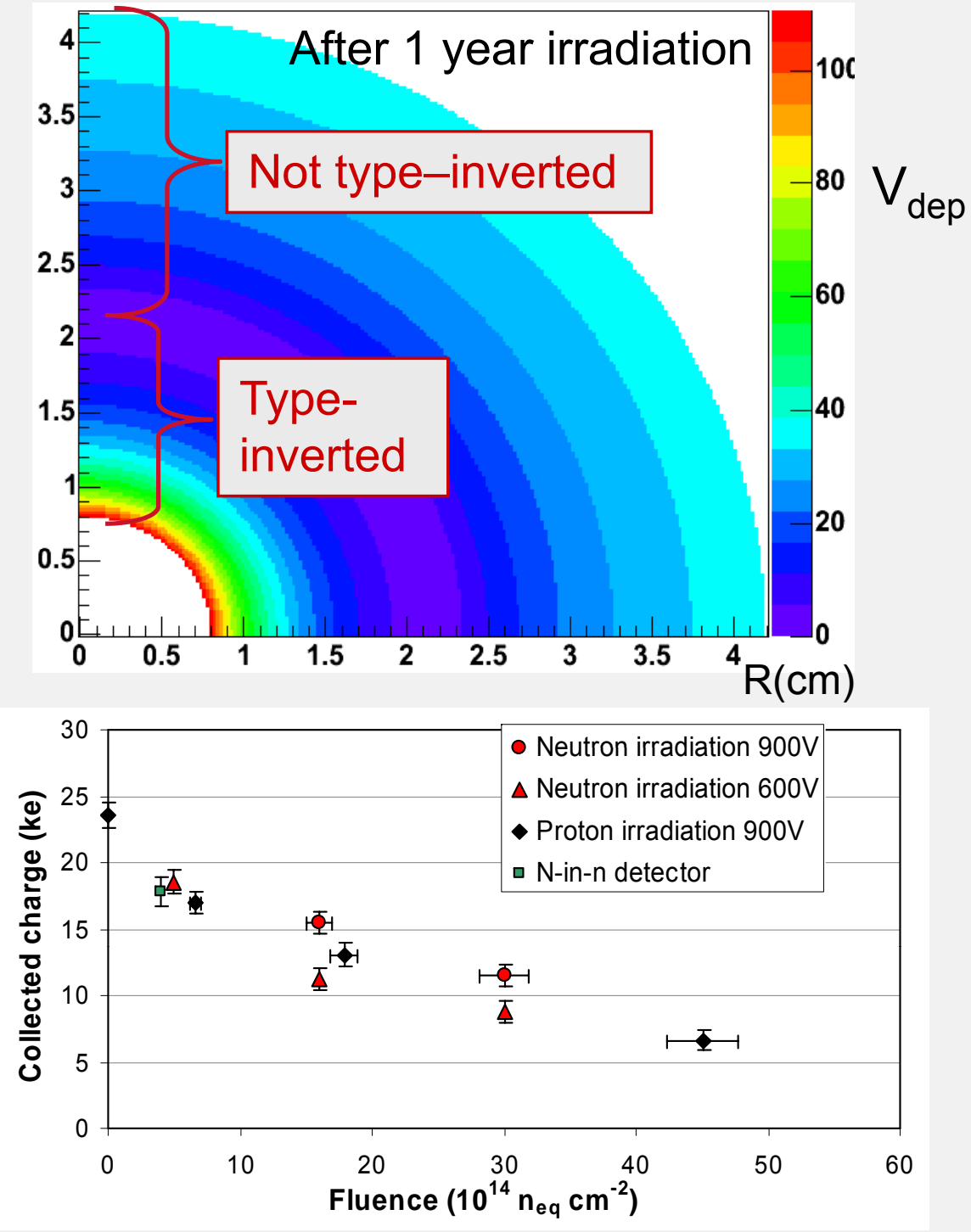


\section{Hybrid Substrates}

-TPG (Thermal Pyrolitic Graphite) core

$>4 \mathrm{x}$ more thermally conductive than copper

$>$ Removes $24 \mathrm{~W}$ of heat with a designed $\Delta \mathrm{T}$ of $20^{\circ} \mathrm{C}$ between coolant and sensors

- $\sim 8^{\circ} \mathrm{C}$ coolant-hybrid,

$-\sim 8^{\circ} \mathrm{C}$ within hybrid

- $\sim 4^{\circ} \mathrm{C}$ hybrid-to-sensor

- Clad in woven Carbon fibre for rigidity
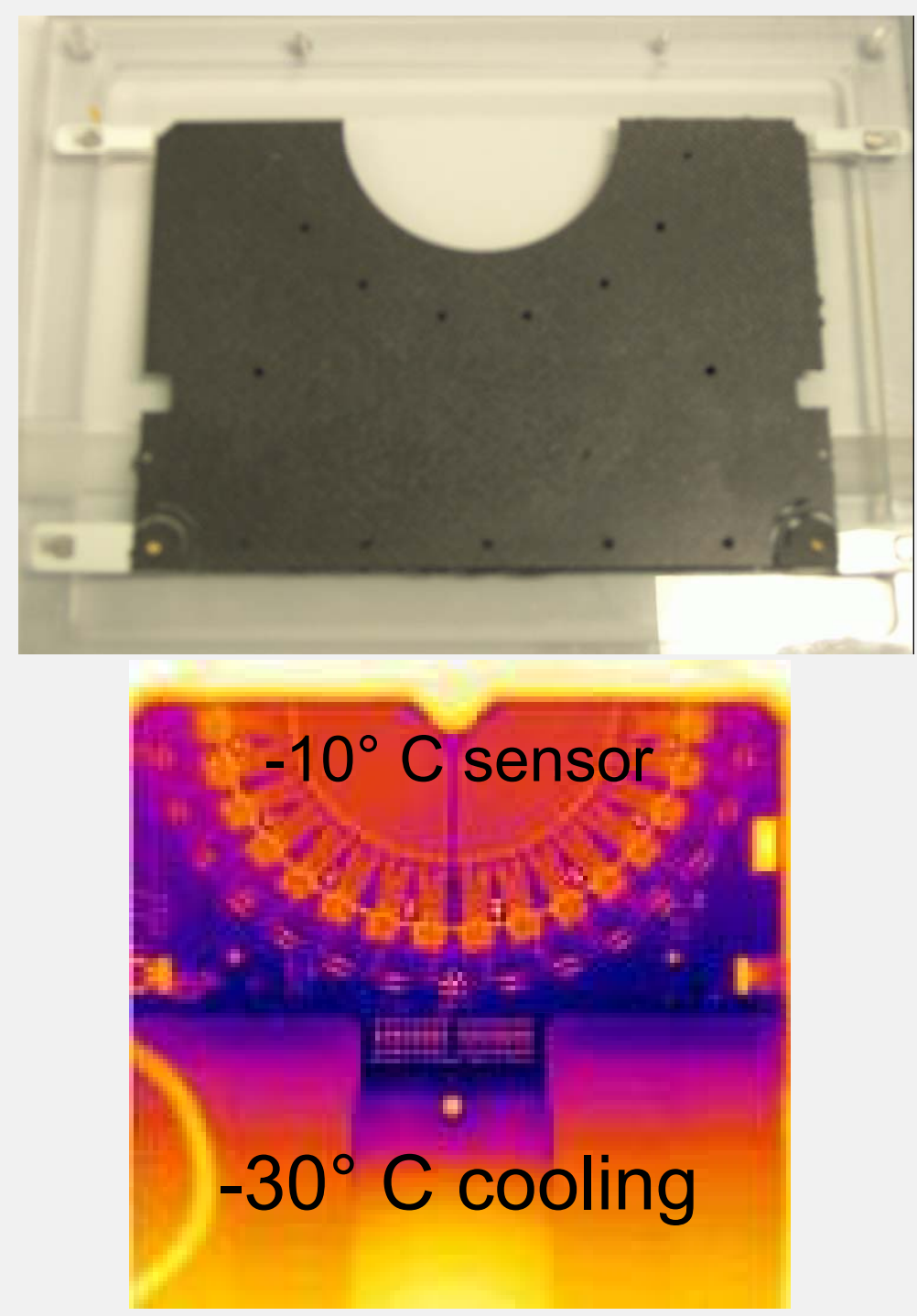


\section{Hybrids}
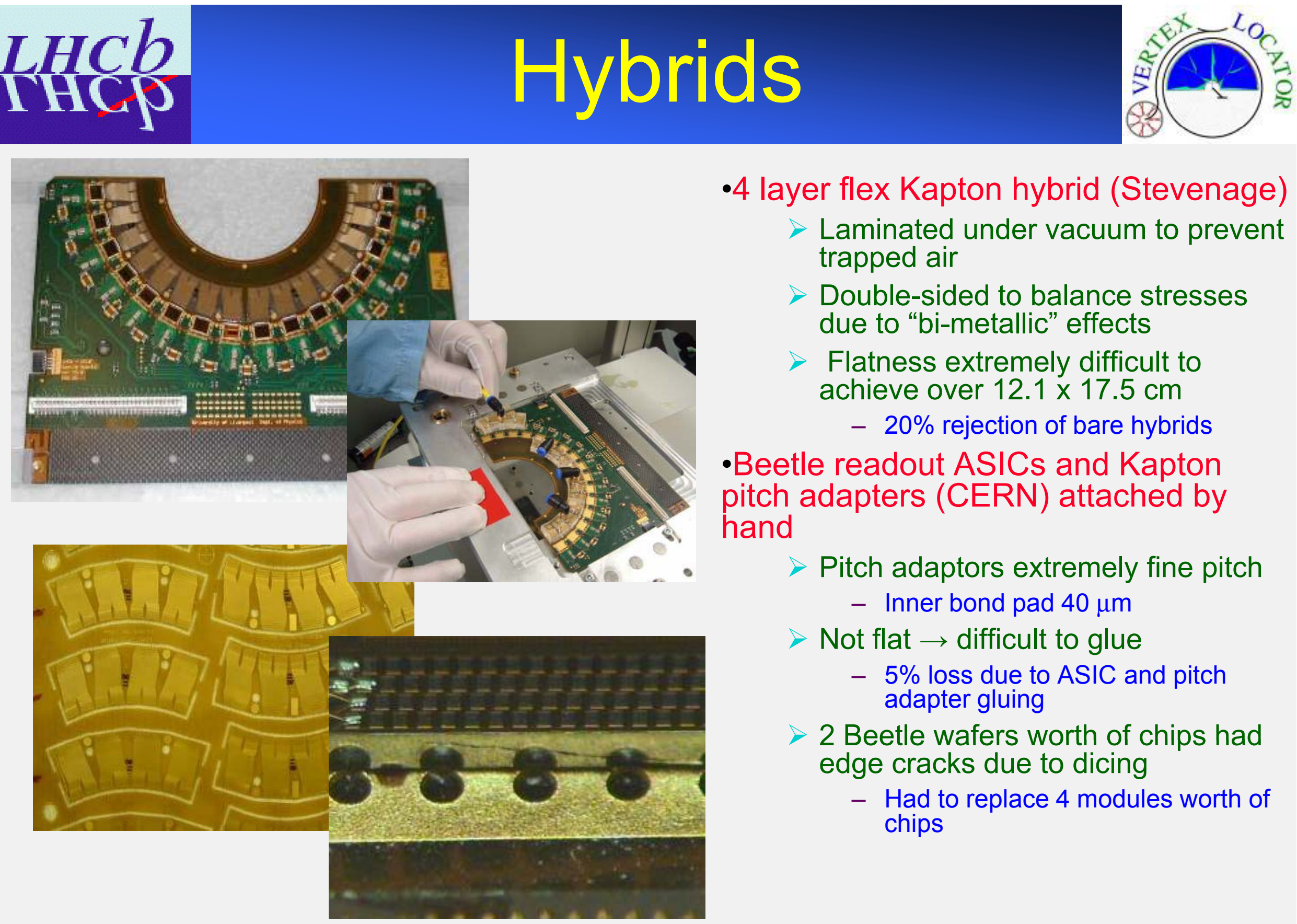

-4 layer flex Kapton hybrid (Stevenage)

$>$ Laminated under vacuum to prevent trapped air

$>$ Double-sided to balance stresses due to "bi-metallic" effects

$>$ Flatness extremely difficult to achieve over $12.1 \times 17.5 \mathrm{~cm}$

- $20 \%$ rejection of bare hybrids

-Beetle readout ASICs and Kapton pitch adapters (CERN) attached by hand

$>$ Pitch adaptors extremely fine pitch

- Inner bond pad $40 \mu \mathrm{m}$

$>$ Not flat $\rightarrow$ difficult to glue

- $5 \%$ loss due to ASIC and pitch adapter gluing

$>2$ Beetle wafers worth of chips had edge cracks due to dicing

- Had to replace 4 modules worth of chips 


\section{Wirebonding}
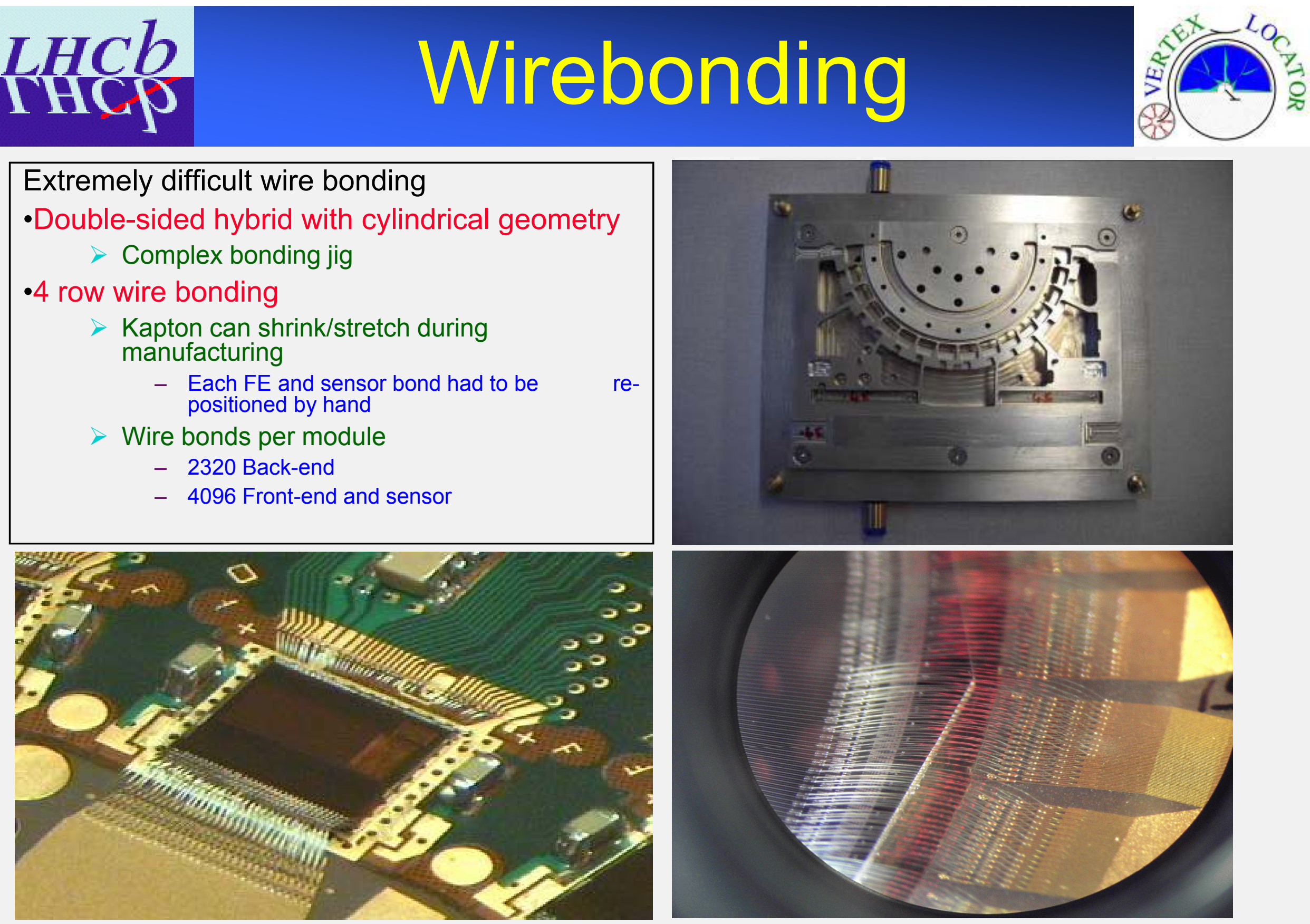

RD07, Firenze, June 27, 2007

LHCb VELO Module Production and Performance -Anthony Affolder

Slide 9 
-Problems encountered

$>$ Hybrid movement during bonding- took 1 year to remove

$>$ Vibration due to nearby construction (pile-driving)

- H \& K 710 wire bonder severely sensitive to motion

Weak wire bonds

- Purchased seismometer (Apple Power Book) to monitor motion

$>$ Smallest bonding pads on Kapton pitch adaptor over-etched to $20-25 \mu \mathrm{m}$

-Average time for bonding of 3 man-days per module

$>$ But no module failures due to bonding and only $0.3 \%$ extra faulty channels introduced

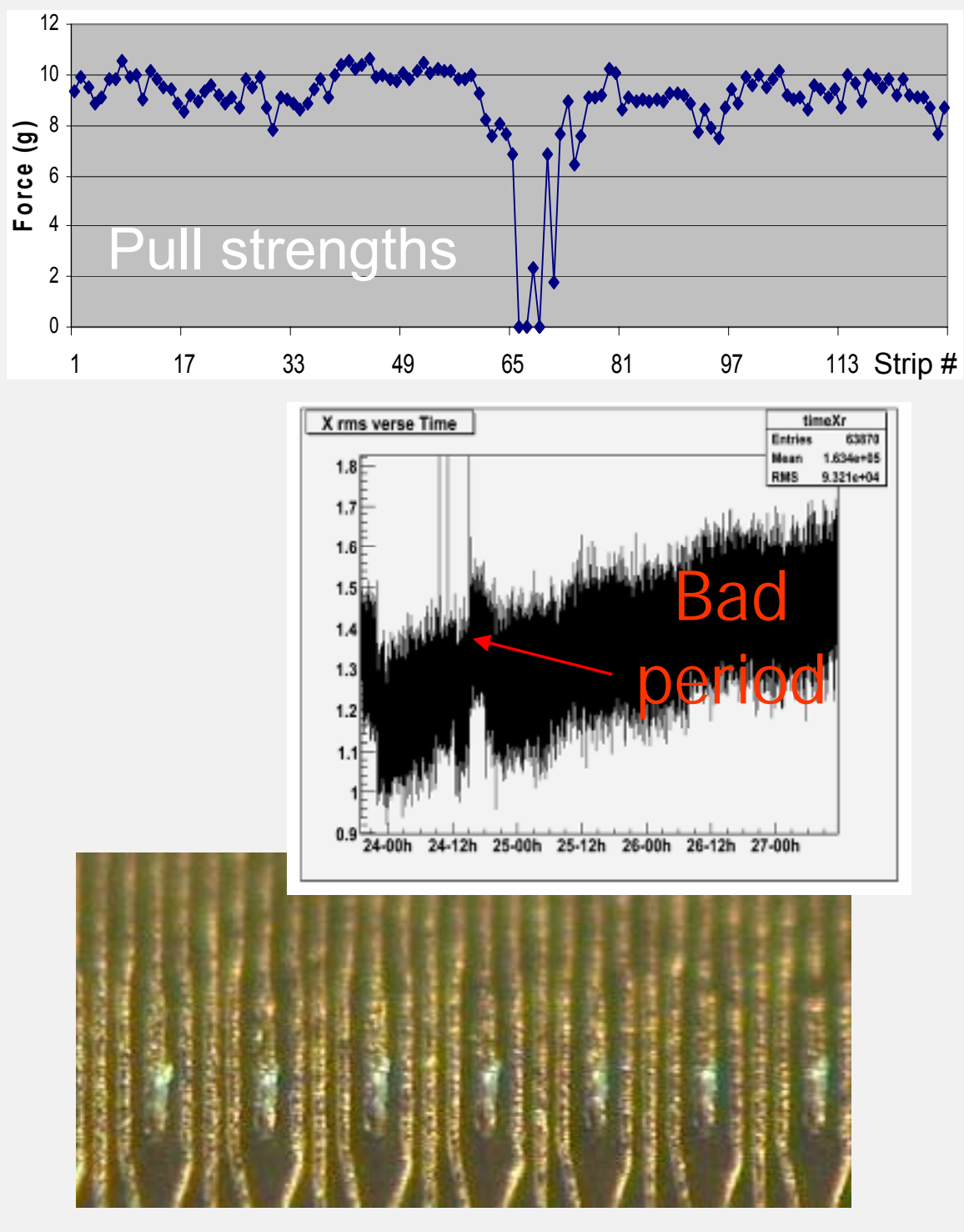



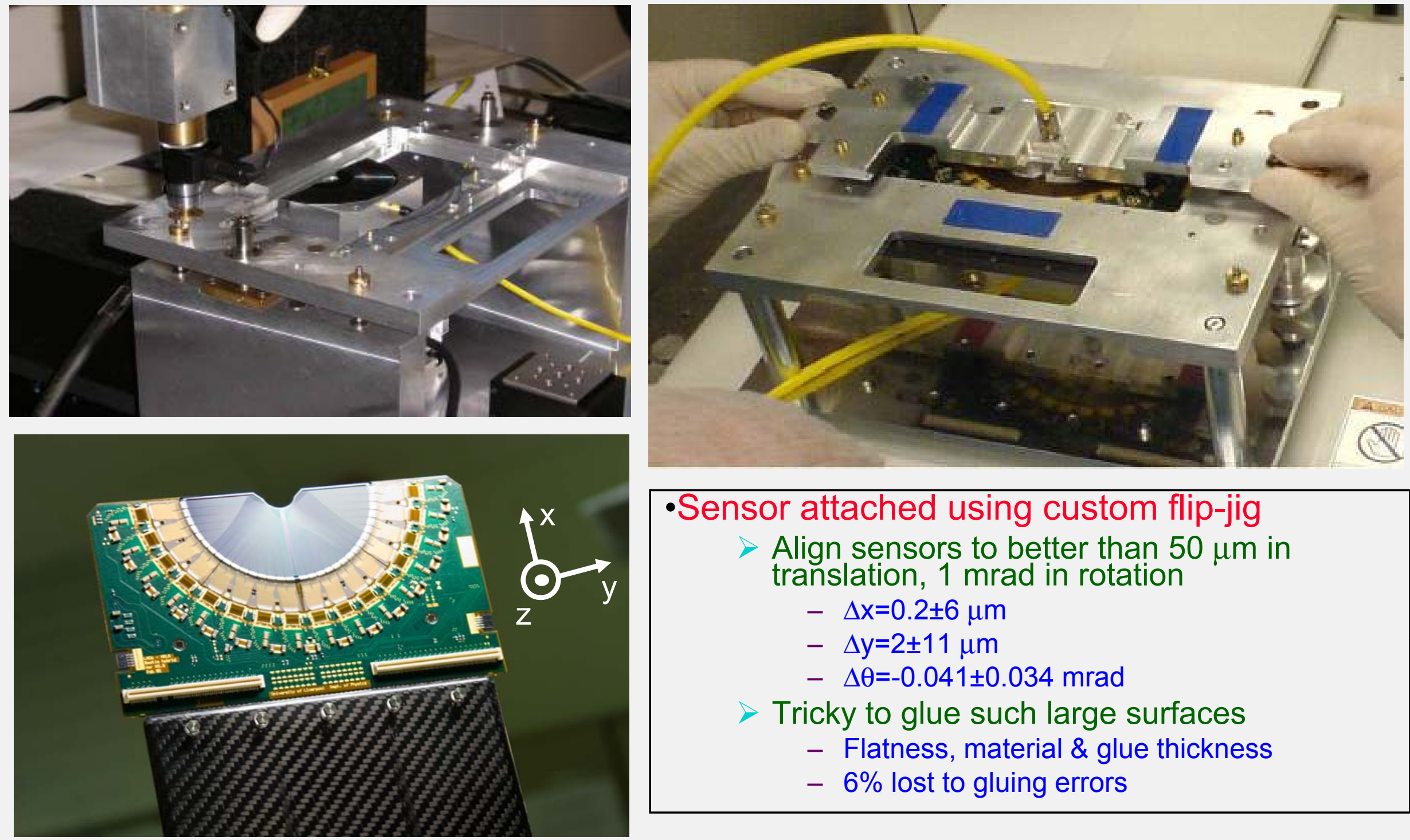

-Sensor attached using custom flip-jig

$>$ Align sensors to better than $50 \mu \mathrm{m}$ in translation, $1 \mathrm{mrad}$ in rotation

$-\Delta \mathrm{x}=0.2 \pm 6 \mu \mathrm{m}$

- $\Delta \mathrm{y}=2 \pm 11 \mu \mathrm{m}$

- $\Delta \theta=-0.041 \pm 0.034 \mathrm{mrad}$

$>$ Tricky to glue such large surfaces

- Flatness, material \& glue thickness

- $6 \%$ lost to gluing errors 
-After each bonding step, the hybrids are electrically tested

$>$ Pedestal, noise, and laser (with sensors)

-Opens and shorts easily found

$>$ But pinholes impossible to see

- Full laser signal with only $20 \%$ increase in noise when inducing a bias current of $5 \mathrm{~mA}$ with light

- Beetle chip could probably be used with DC coupled sensors

-Found 3 sensors ( $6 \%$ of modules) with problems with $\mathrm{p}$-spray isolation

$>$ Was not possible to test for during probing with current sensor design
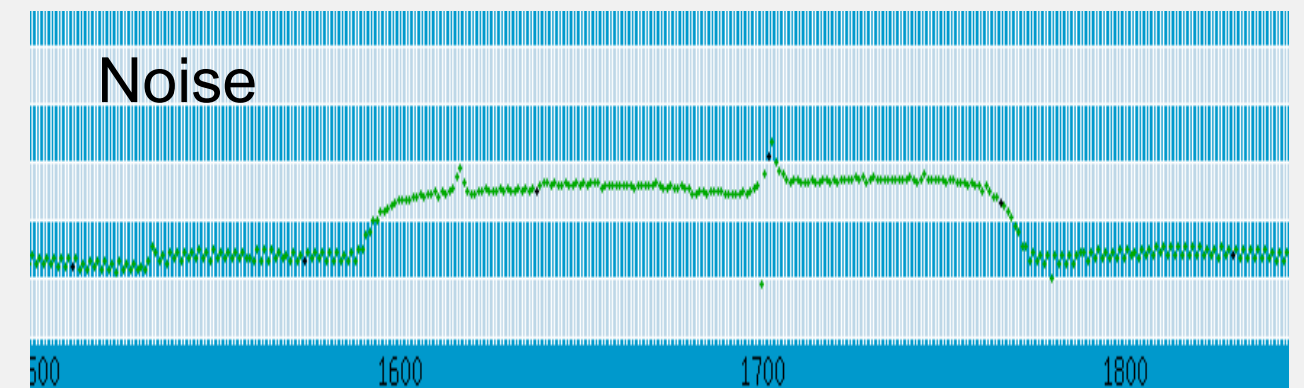
1700 1800

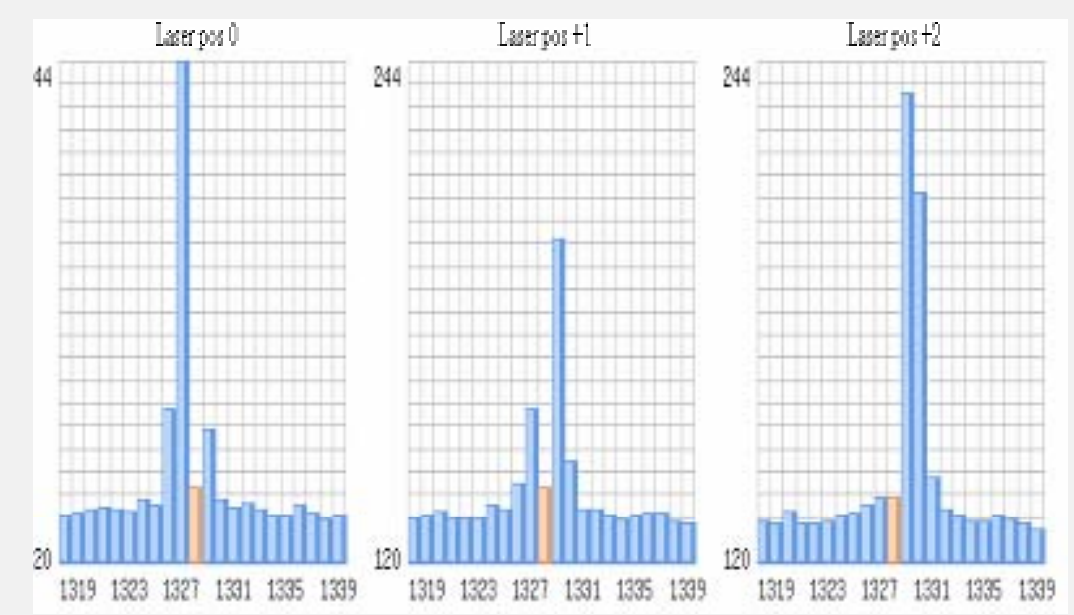




\section{Pedestals}

-Hybrids mounted to VELO base on carbon-fibre pedestals

$>\sim 0$ CTE

$>$ Manufactured in-house to avoid air volumes

-Hybrid glued to pedestal with Smartscope system

$>$ For trigger, $\mathrm{R}$ sensor aligned to $40 \mu \mathrm{m}$ translation, $1 \mathrm{mrad}$ rotation relative to pedestal base pin
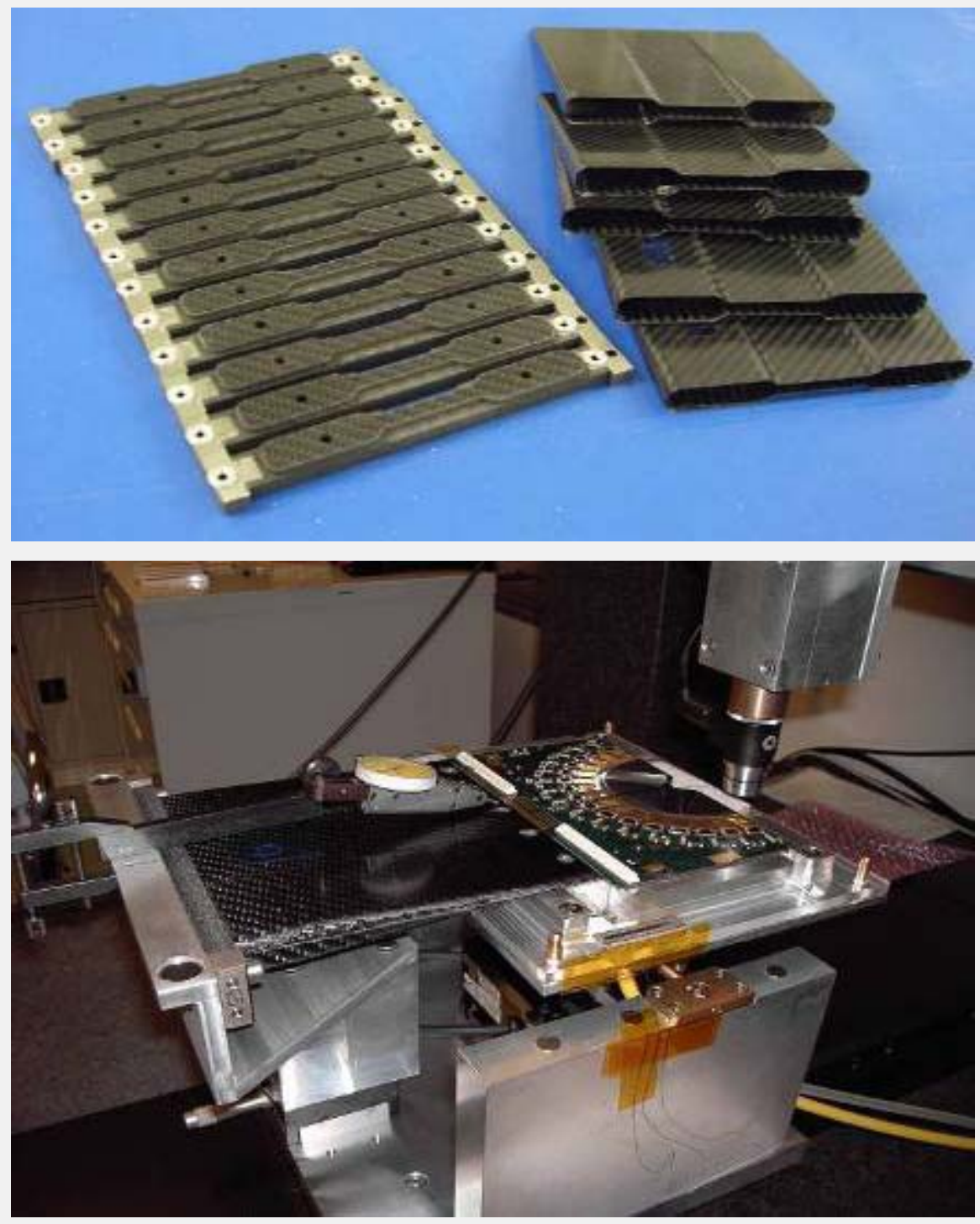


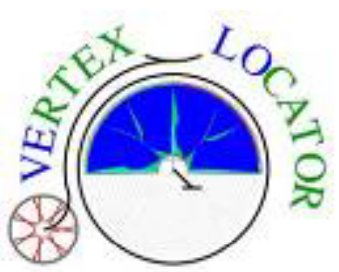

-Each module measured on assembly, on cable attachment, and after vacuum testing on CMM

$>$ R-sensor (in trigger):

- $\Delta \mathrm{x}=-0.4 \pm 9 \mu \mathrm{m}, \Delta \mathrm{y}=3 \pm 13 \mu \mathrm{m}$ $\Delta \theta=-0.072 \pm 0.131 \mathrm{mrad}$

$>$ Phi-sensor:

- $\Delta \mathrm{x}=-2 \pm 8 \mu \mathrm{m}, \Delta \mathrm{y}=5 \pm 18 \mu \mathrm{m}$ $\Delta \theta=-0.067 \pm 0.141 \mathrm{mrad}$

$>$ But translation along beam difficult (44\% outside of $\pm 200 \mu m$ specification)
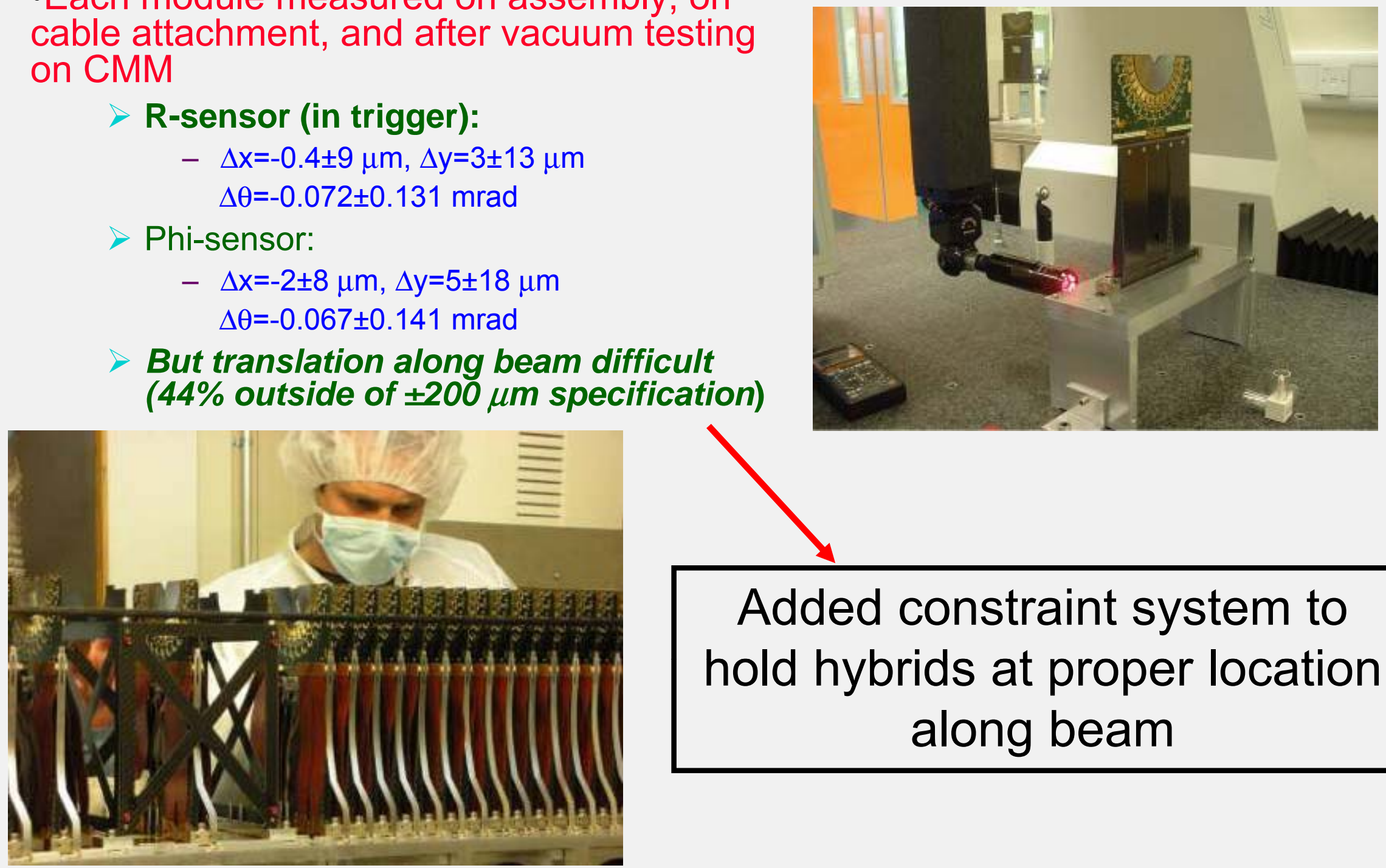

Added constraint system to hold hybrids at proper location along beam 
-All modules tested in vacuum tank with near final $\mathrm{CO}_{2}$ cooling system and $\mathrm{DAQ}$

$>\sim 1 \times 10^{-3}$ mbar with coolant at $-30^{\circ} \mathrm{C}$

-Electrical tests confirmed previous faulty channel lists

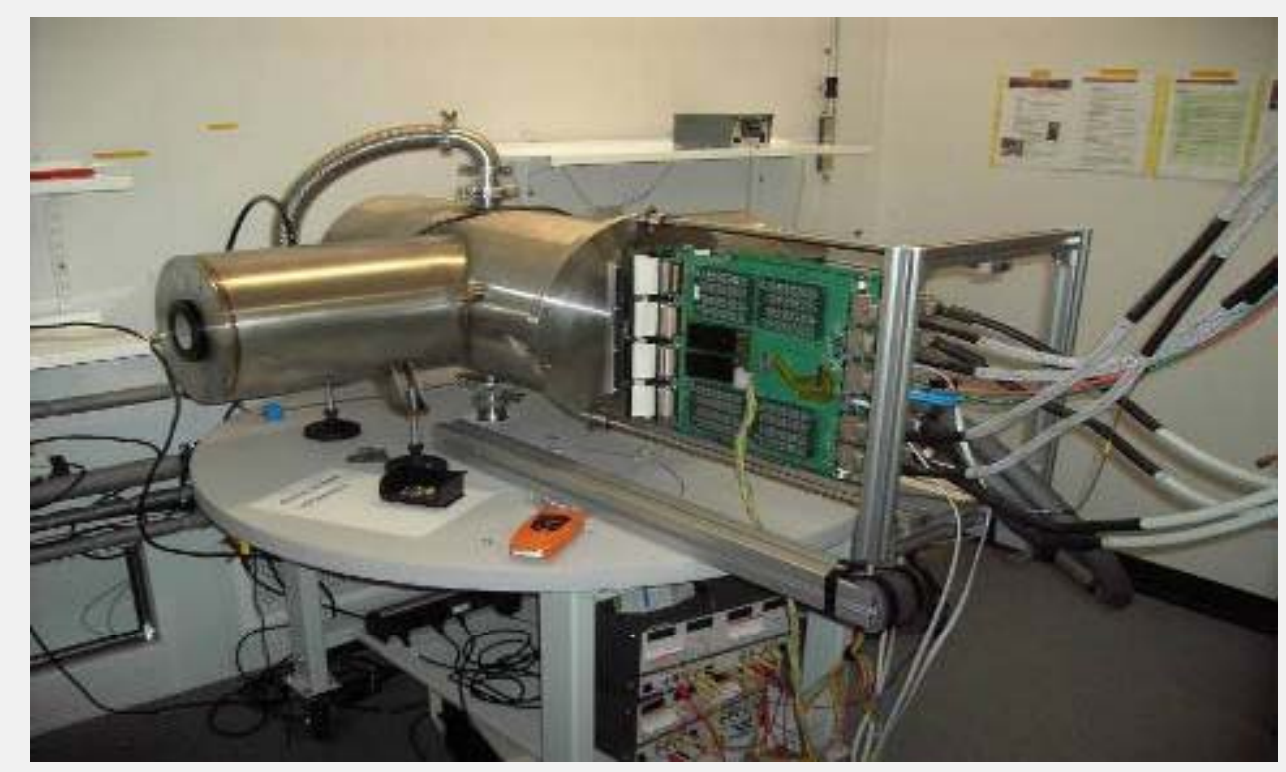

-Thermal performance as expected

$>\Delta \mathrm{T}=-22.8^{\circ} \mathrm{C}$ between coolant and sensor

- Should be $2-3^{\circ} \mathrm{C}$ less with cold neighbours

$>2$ modules had anomalous cooling performance and were rejected $(4 \%)$

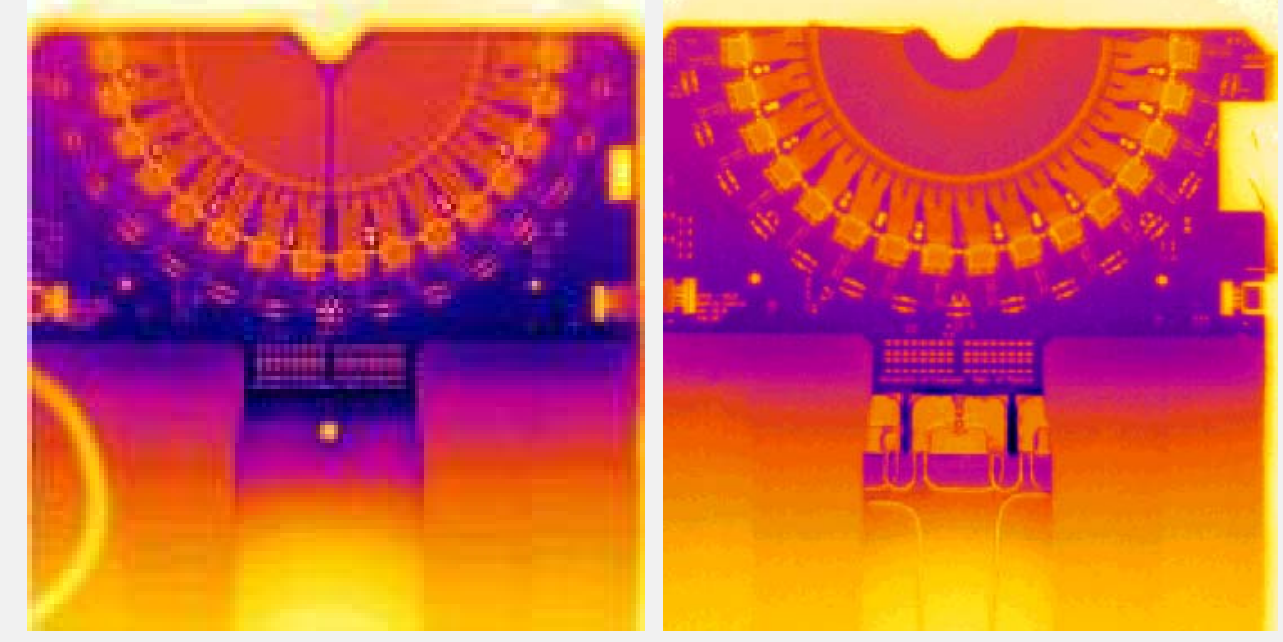


IHCb Reception/Burn-in@CERN Oి

-Every module visually re-inspected on arrival at CERN

$>3$ hrs per module

-Module Burn-in

$>$ Electrical tests in vacuum $\left(10^{-6} \mathrm{mbar}\right)$

- Noise, pedestals, bias currents

$>$ Thermal stressing

- 4 cycles between $-30^{\circ} \mathrm{C}$ and $30^{\circ} \mathrm{C}$

$>$ Electronics burn-in

- >16 hrs at $30^{\circ} \mathrm{C}$
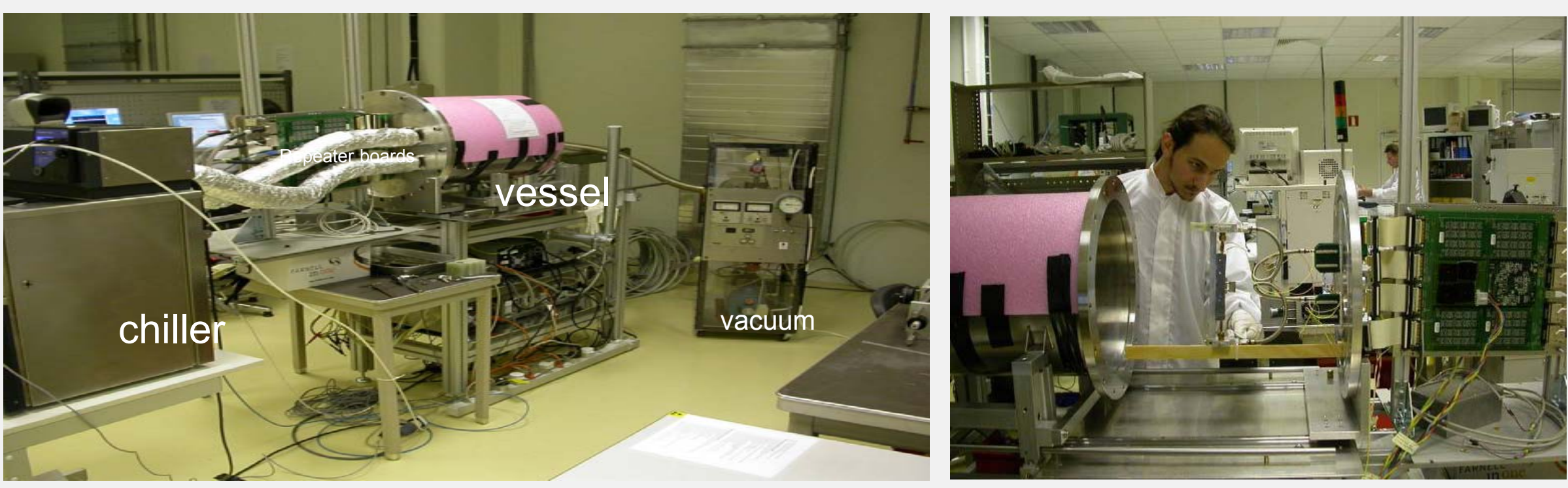
- Found damage to bias return wire bonds

$>$ Required emergency epoxy fix on first few modules

- Great stability of module performance

$>$ No additional opens/shorts

$>$ No ASICs failures

$>$ Only 1 sensor showed significant bias current increase during burn-in

- Stable for over 3 days
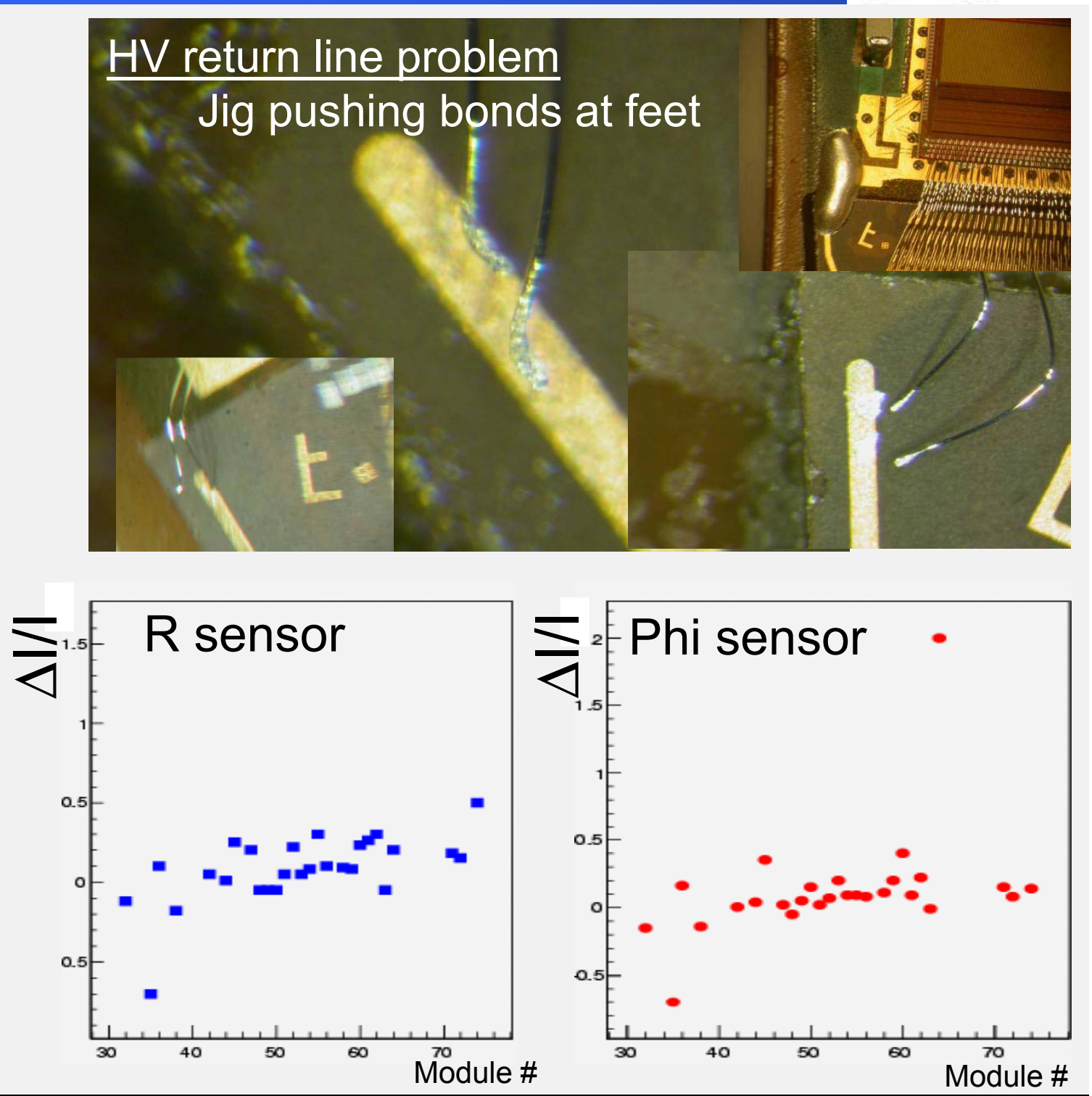

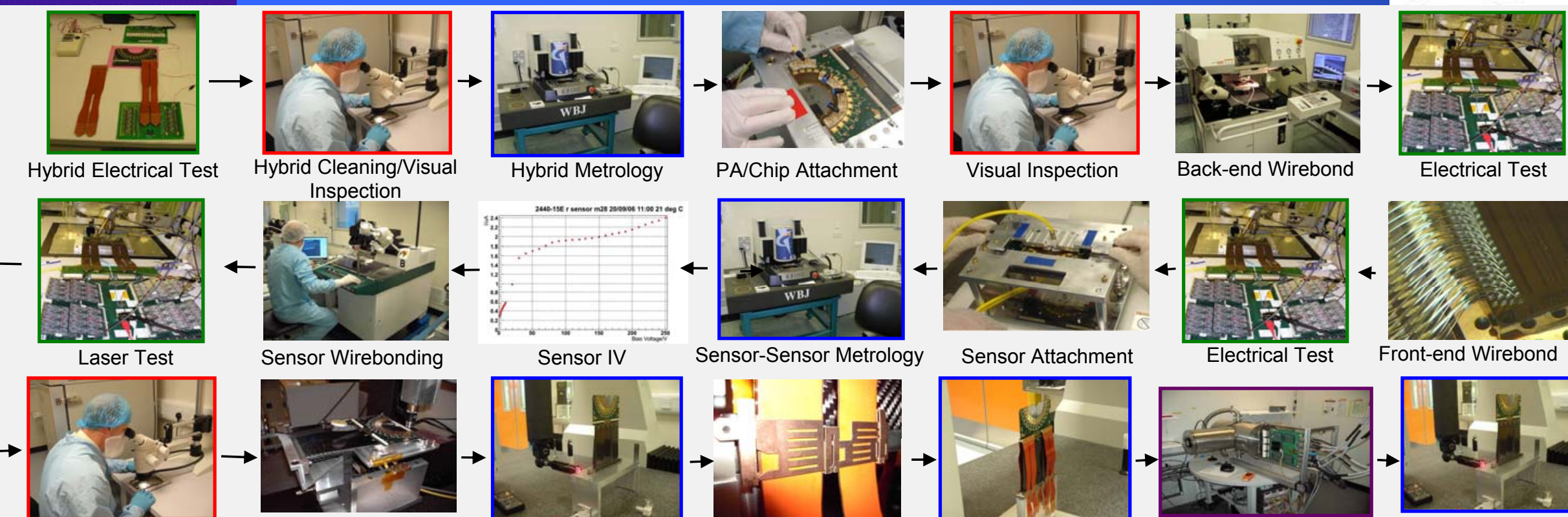

Hybrid Cleaning/Visual

PA/Chip Attachment

Visual Inspection

Back-end Wirebond

Electrical Test
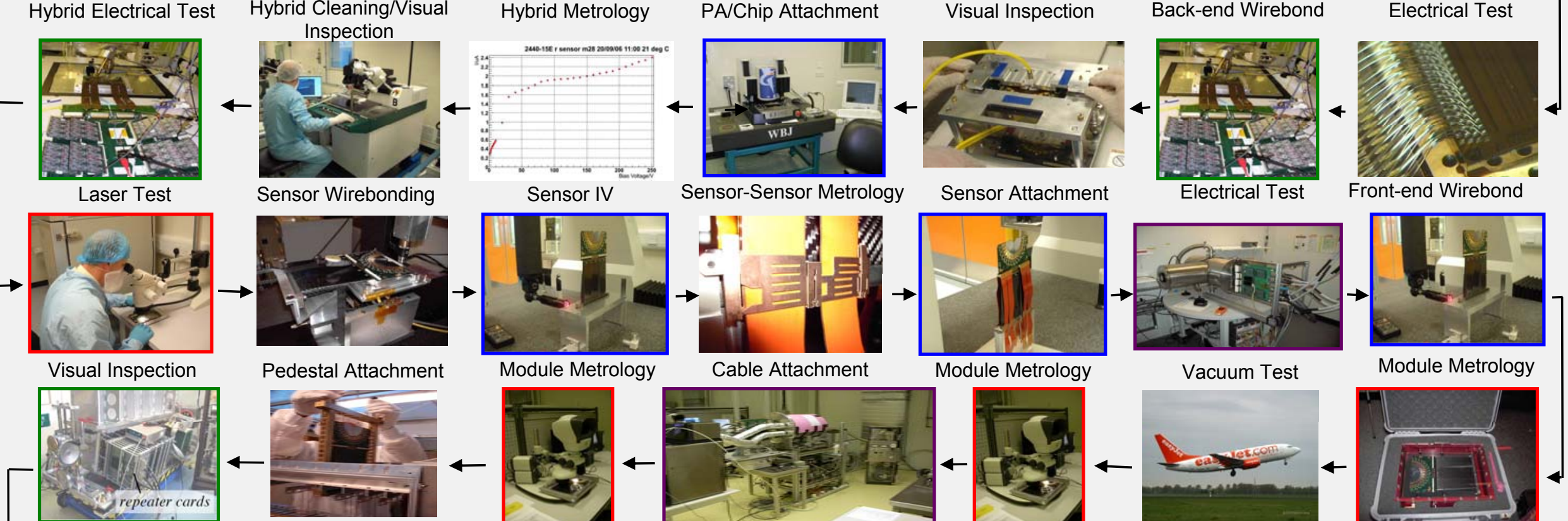

Pedestal Attachment

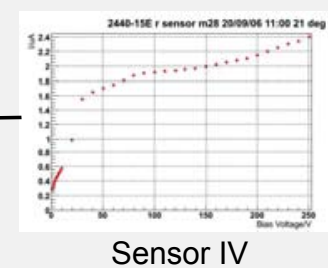

Sensor-Sensor Metrology
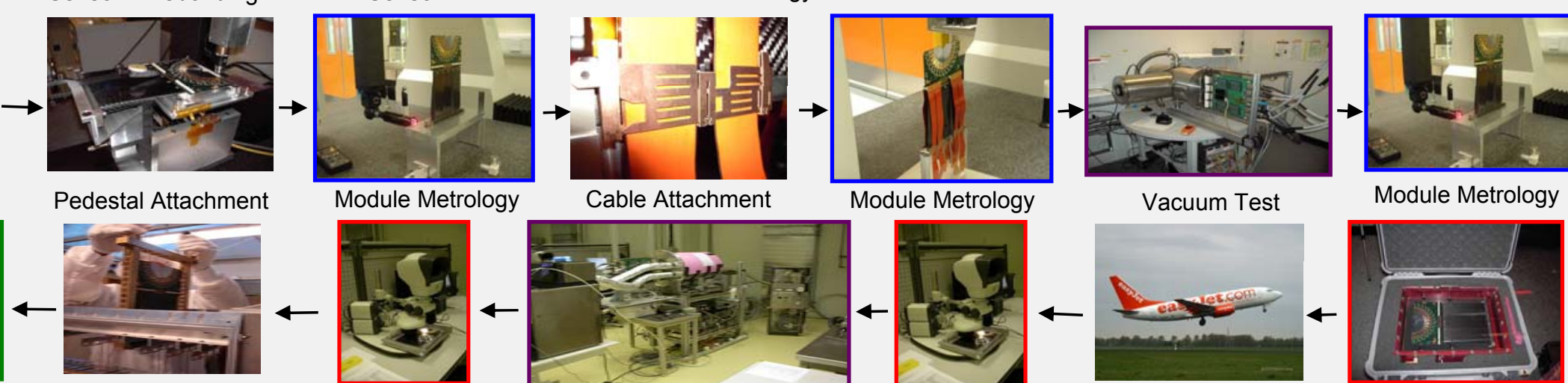

Cable Attachment

Module Metrology

Vacuum Test
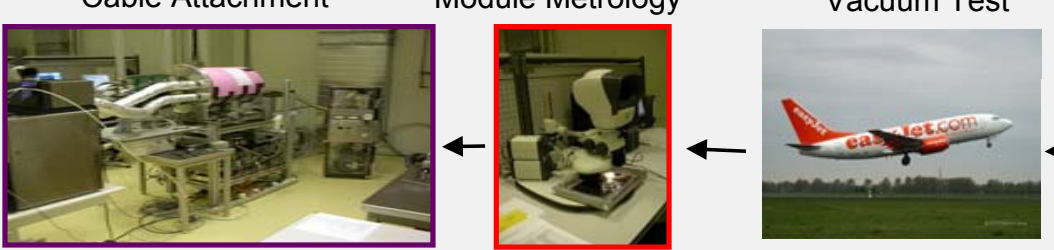

Module Metrology

Electrical Test

Assemble onto VELO half Visual Inspection Module Burn-in

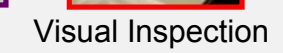

Ship to CERN
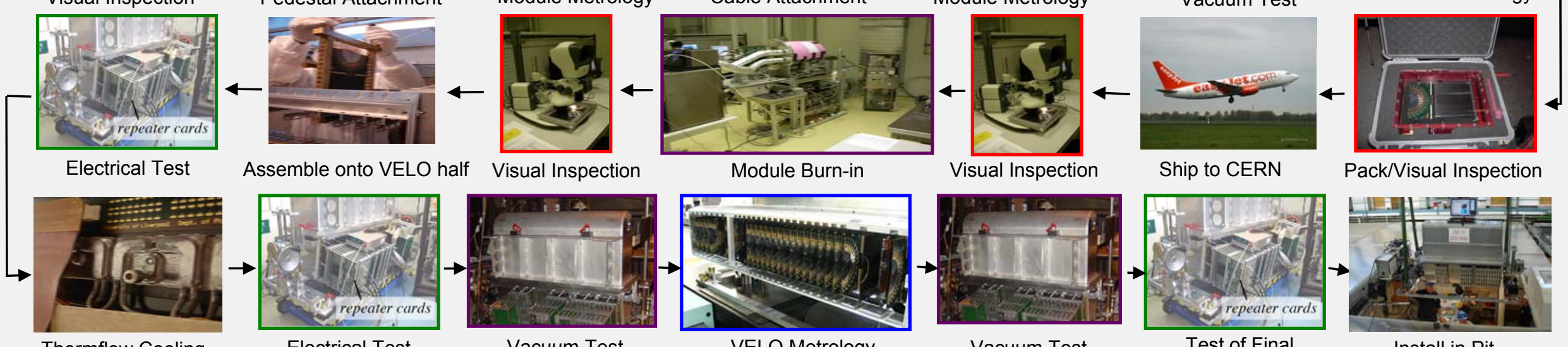

Vacuum Test

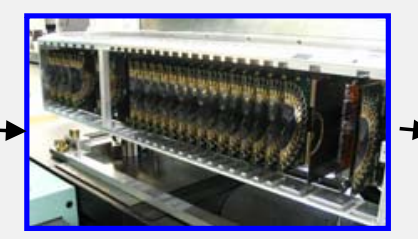

VELO Metrology

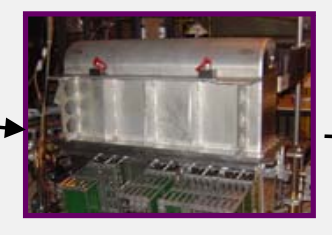

Vacuum Test

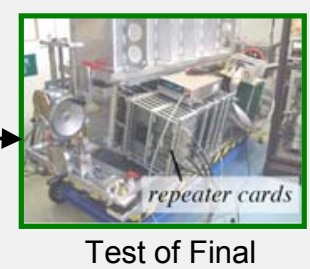

Test of Final

6 Visual Inspections, 6 Metrologies, 7 Electrical Tests, 4 Vacuum Tests

RD07, Firenze, June 27, 2007

LHCb VELO Module Production and Performance -Anthony Affolder

Slide 18

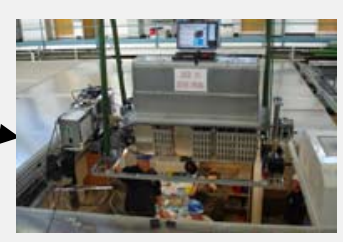

Install in Pit

7

( 


\section{LHCb MACP \\ Final Production Numbers}

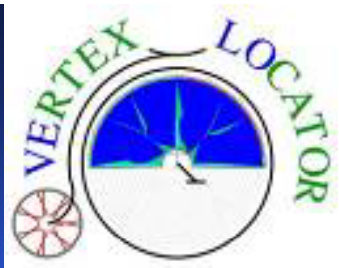

$\neg-$ Delivered $-\square-$ Inspected $-*-$ Mounted $\_$Burnt-in (offset)

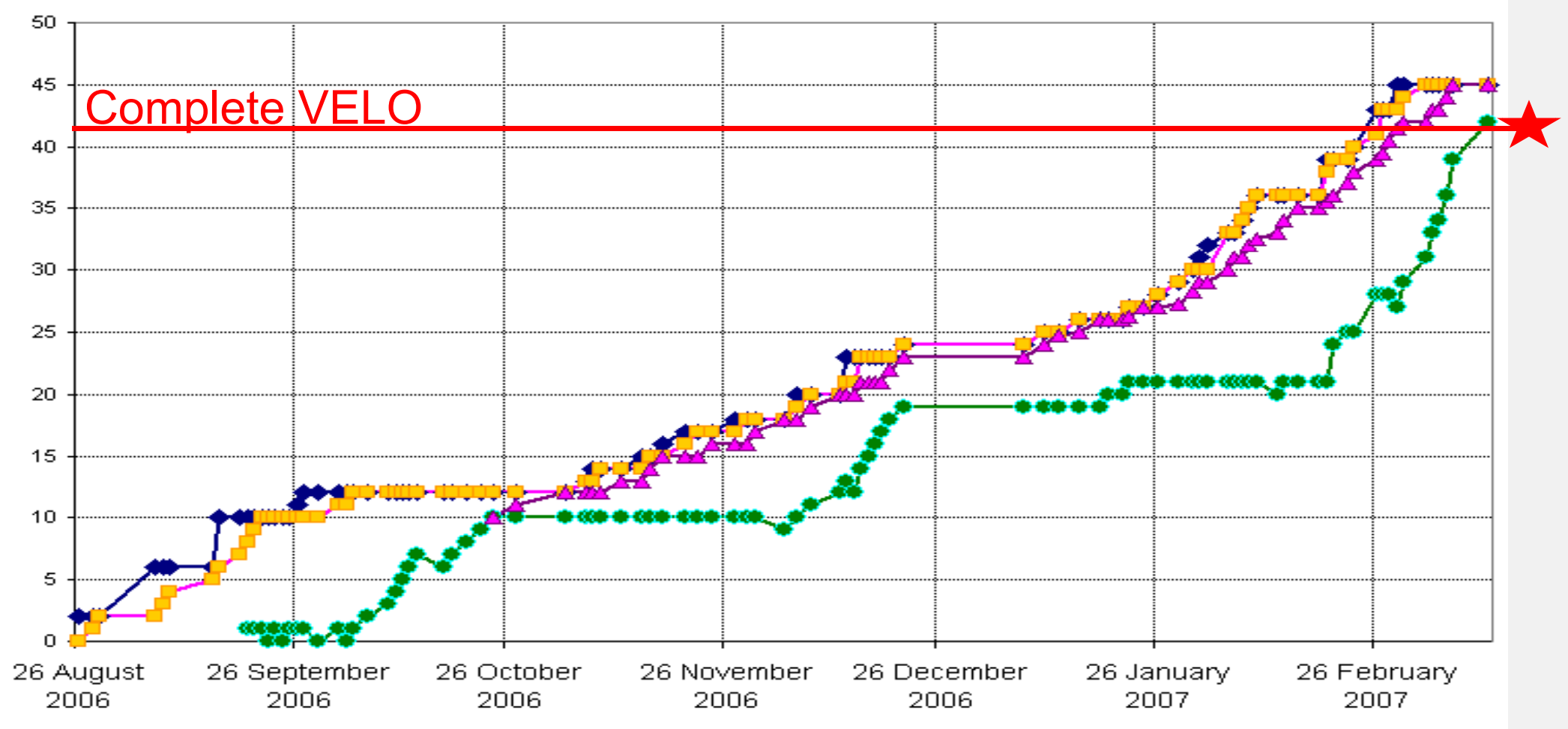

-42 installed modules

$\cdot 0.6 \%$ bad channels per produced over 10 months module

$63 \%$ yield of hybrids

• 100 man-hours per module

$>87 \%$ yield of sensors 


\section{Radiation Thickness}

- Total radiation thickness of system $18.5 \% \mathrm{X}_{0}$

$>$ Largest single source of material is the RF Foil $\left(7.1 \% \mathrm{X}_{0}\right)$

- Effort to reduce/remove foil in upgrades

$>$ Modules $8.1 \% \mathrm{X}_{0}$

- Sensor $4.7 \% X_{0}$

- Hybrid 2.6\% $X_{0}$

- Paddles $0.8 \% X_{0}$
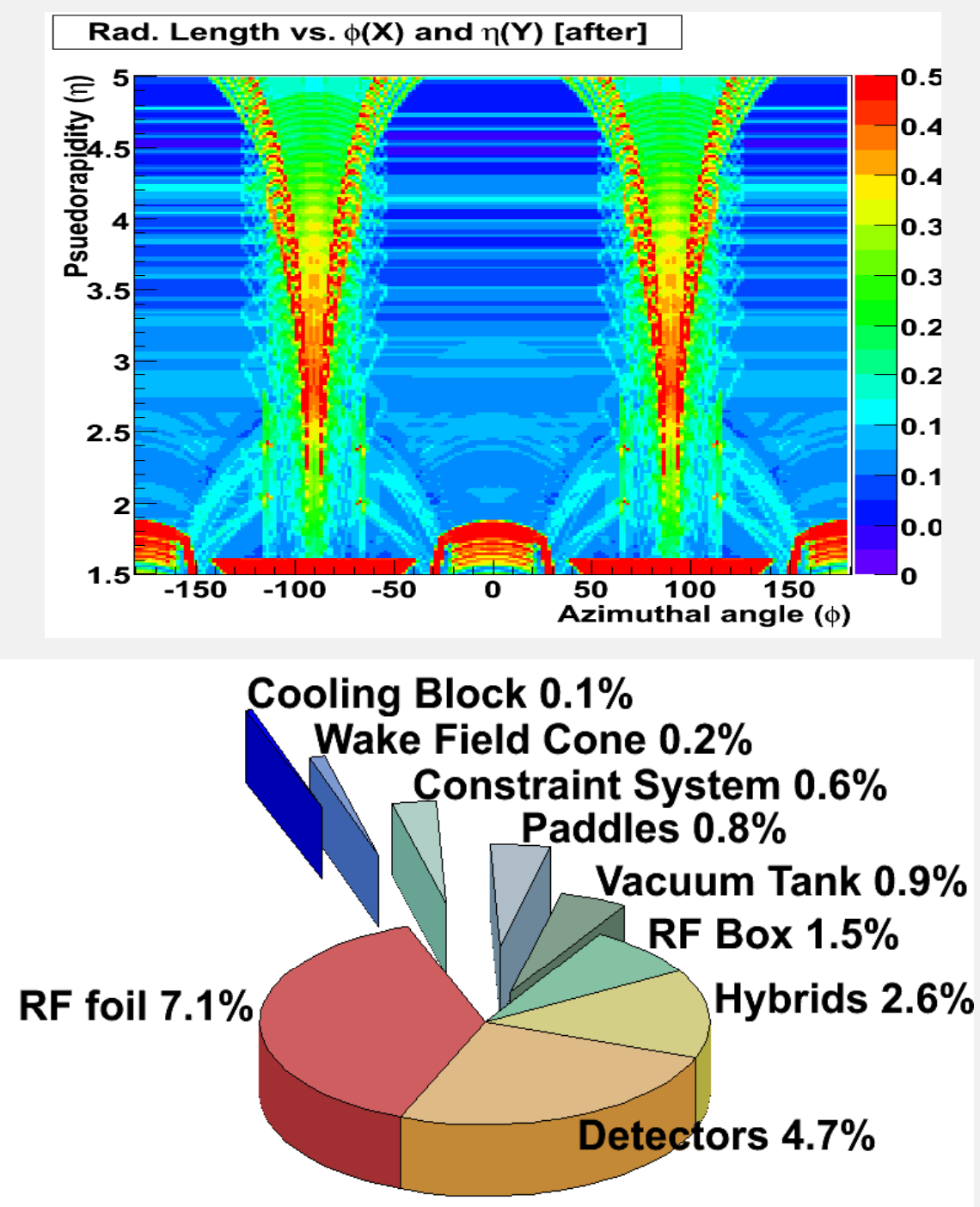


\section{CERN Test Beam}
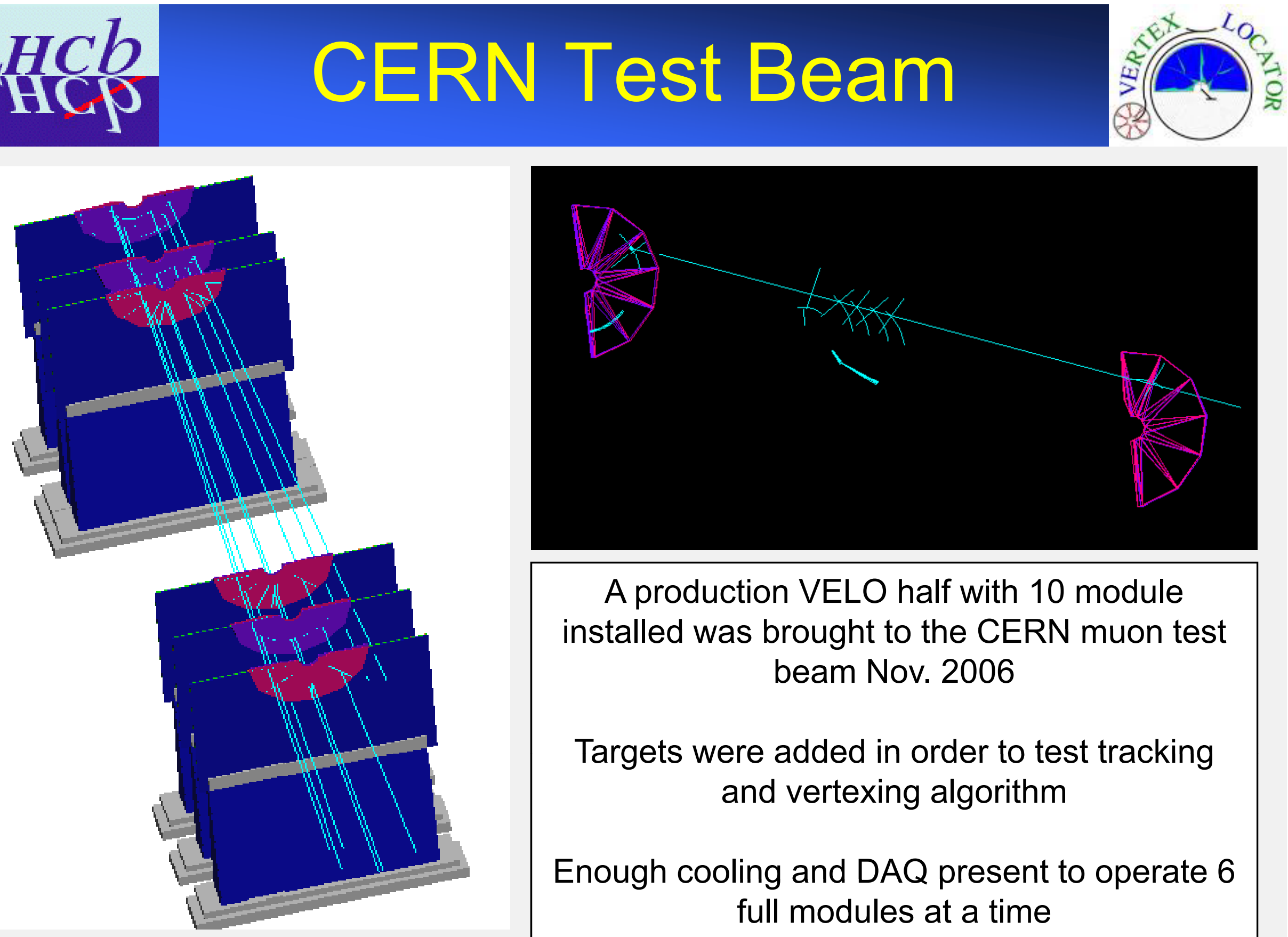

A production VELO half with 10 module installed was brought to the CERN muon test beam Nov. 2006

Targets were added in order to test tracking and vertexing algorithm

Enough cooling and DAQ present to operate 6 full modules at a time 


\section{Module Performance}

(Very) preliminary results of test beam

-Robust signal-to-noise

$>$ Average signal (ADC): $52 \mathrm{R}, 52 \mathrm{phi}$

$>$ Noise varies within sensor due to changing capacitance

- 1.9-2.6 ADC R

- 1.7-2.2 ADC phi

$>$ Signal-to-noise $\sim 20-27$ R, 24-31 phi

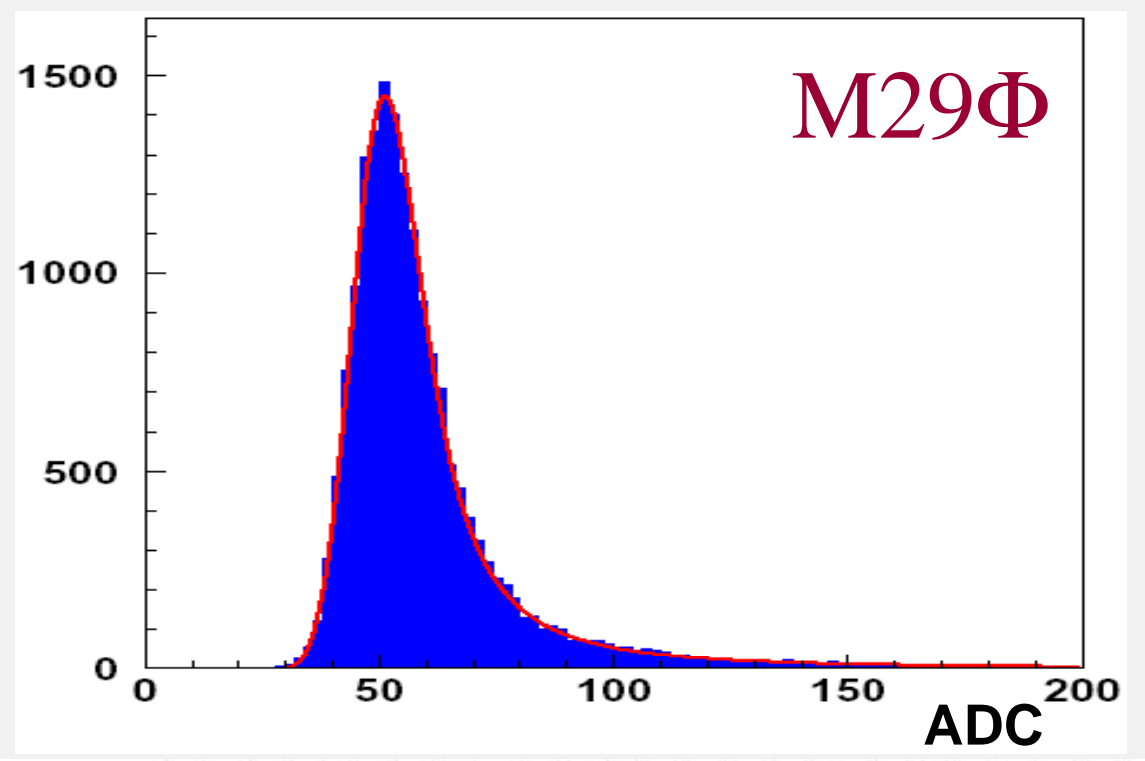

-p bulk detector module shows

expected performance under-depleted

$>\mathrm{n}$ bulk sensors after inversion will behave similarly

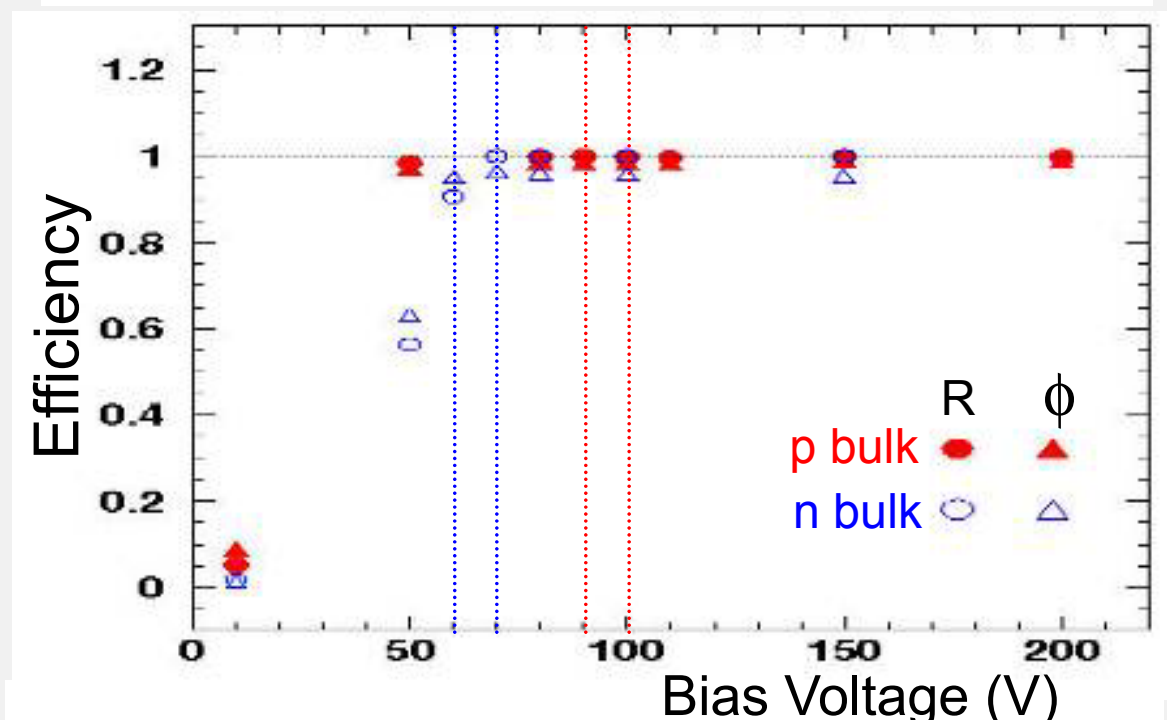




\section{Conclusions}

- VELO module production completed in February 2007

> Some of the most complicated silicon strip detectors ever built

$>$ Mechanical and electrical performance as expected

- Commissioning starting now in the pit with data-taking in 2008

- In near future, we hope to build a complete spare VELO made with $p$ bulk sensors

$>$ Quick replacement in case of beam accident

$>$ Hopefully guarantees full functionality until end of LHC run 


\section{$L H C b$ THCP \\ Noise Performance}

\section{PHI SENSOR}

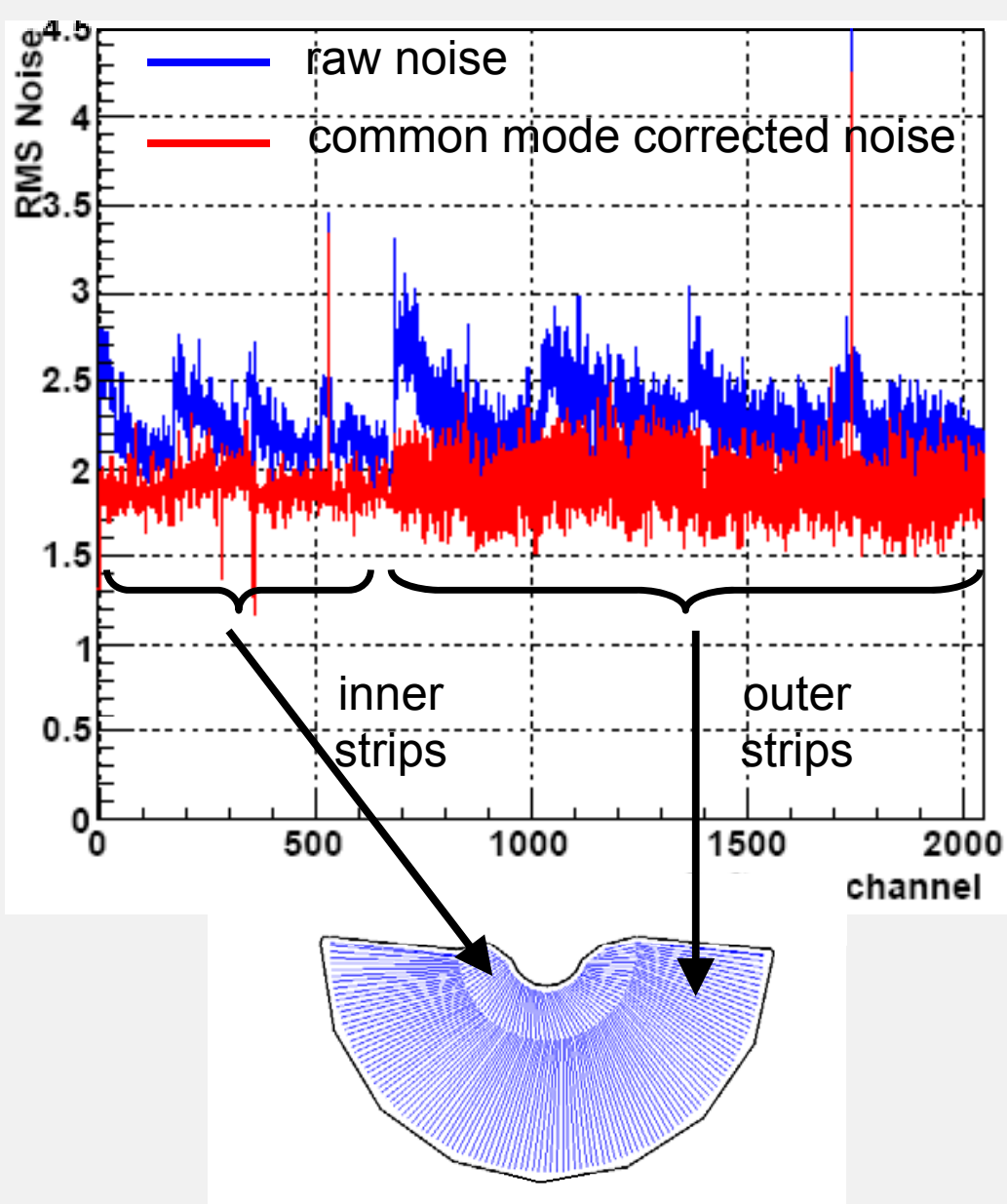

R SENSOR

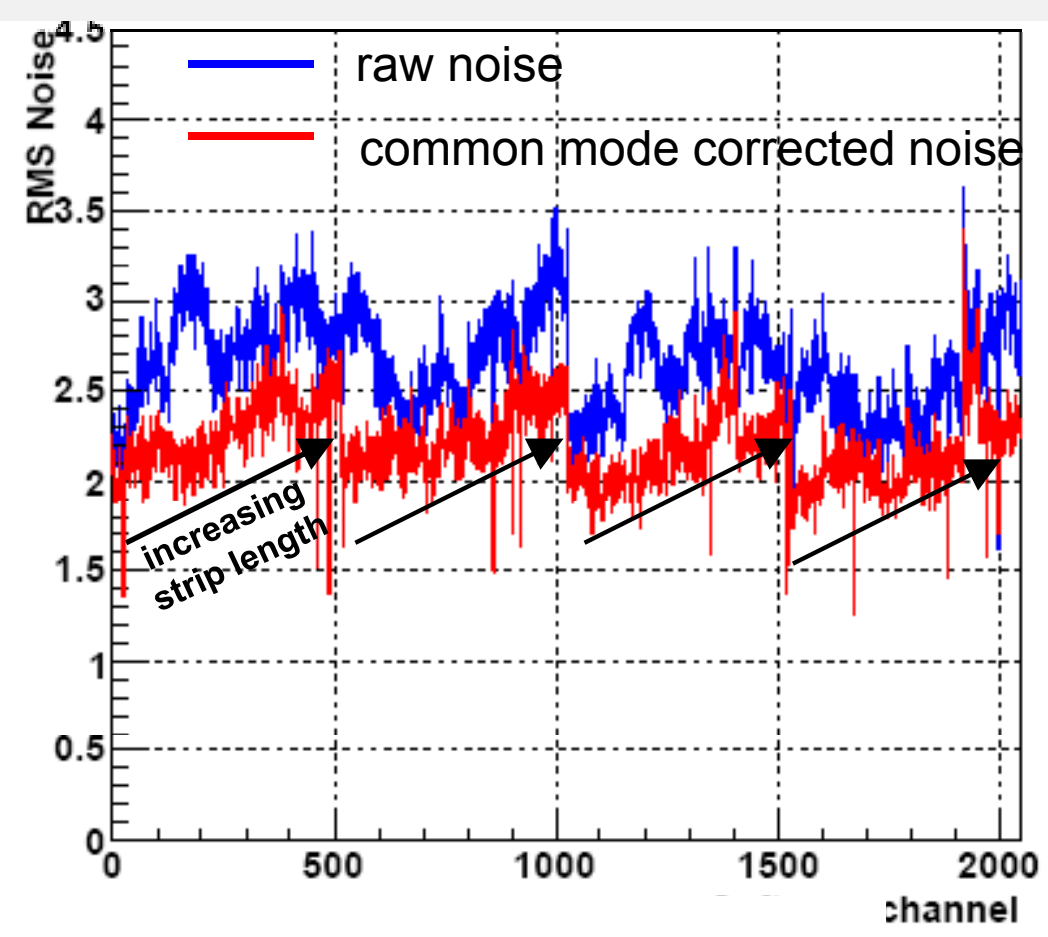




\section{Noise Performance}

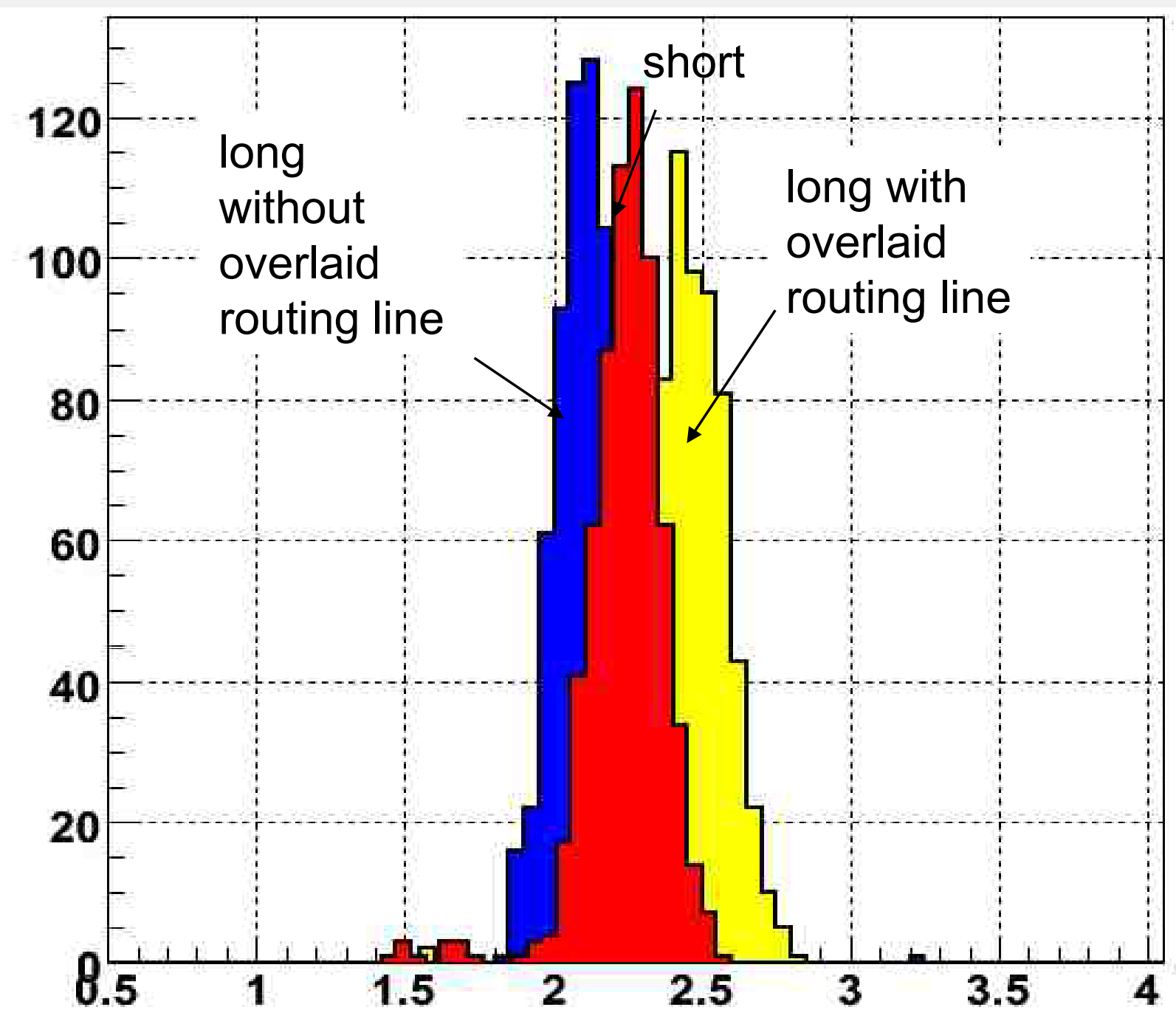




\section{Component Testing}

\begin{tabular}{|c|}
$\bullet$ All components are tested on arrival from vendors \\
$>$ Sensors \\
- Probe station measurements of IV, CV, strip \\
$\quad$ capacitance \\
- Smartscope measurement of size \\
$>$ Pitch Adaptors \\
- Probe station measurement of strip capacitance \\
$>$ Cables \\
- Resistances, opens, shorts \\
$>$ Hybrids (bare \& with surface mounts) \\
- Connectivity, shorts, temperature sensors
\end{tabular}
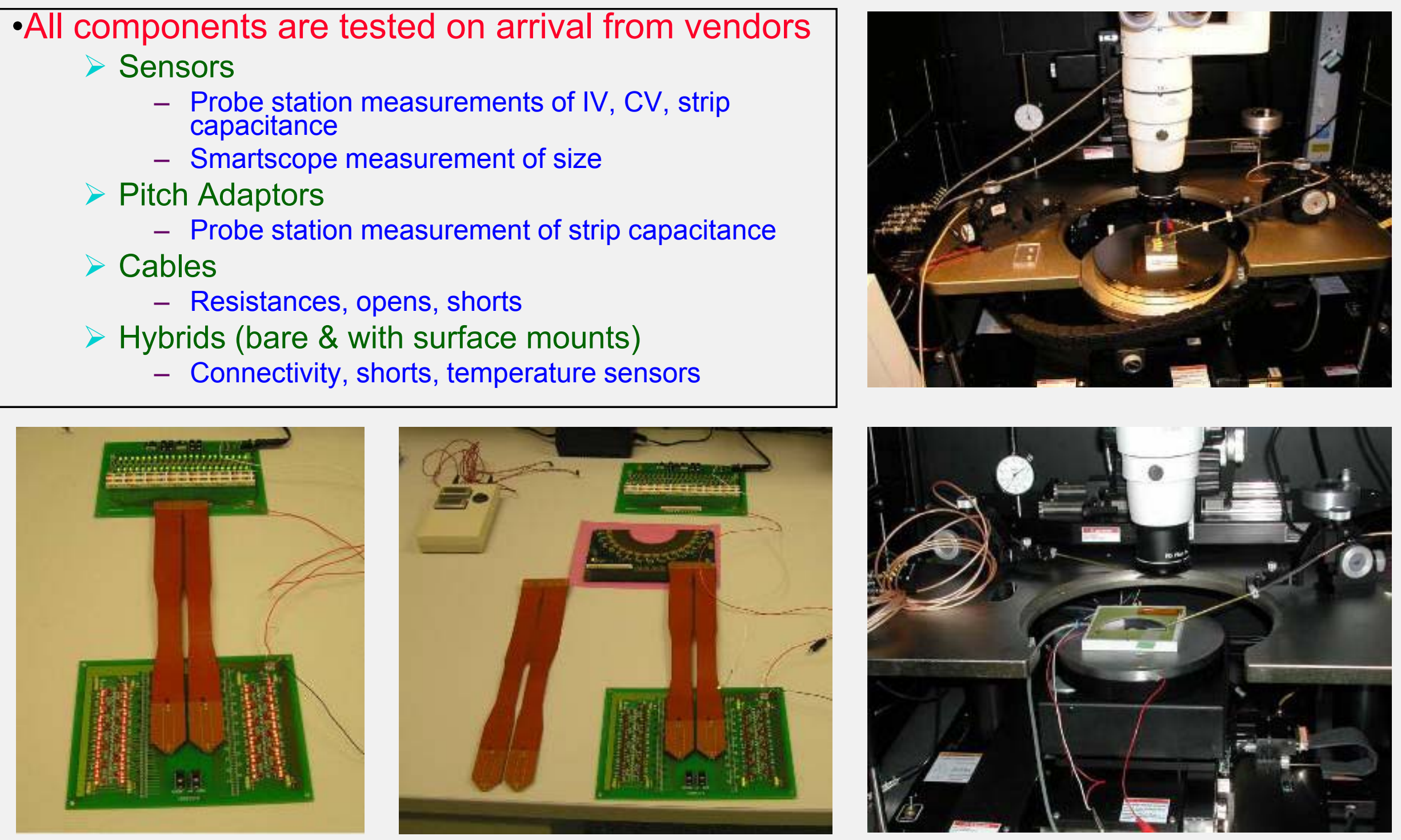


\section{Module Materials}

-Detailed assay of all materials in module made

- Module mass: $321.3 \mathrm{~g}$

- With cables: $406.9 \mathrm{~g}$

$>$ Heavy elements are a concern for activation

$>$ Activation under study

- May impact repair strategy

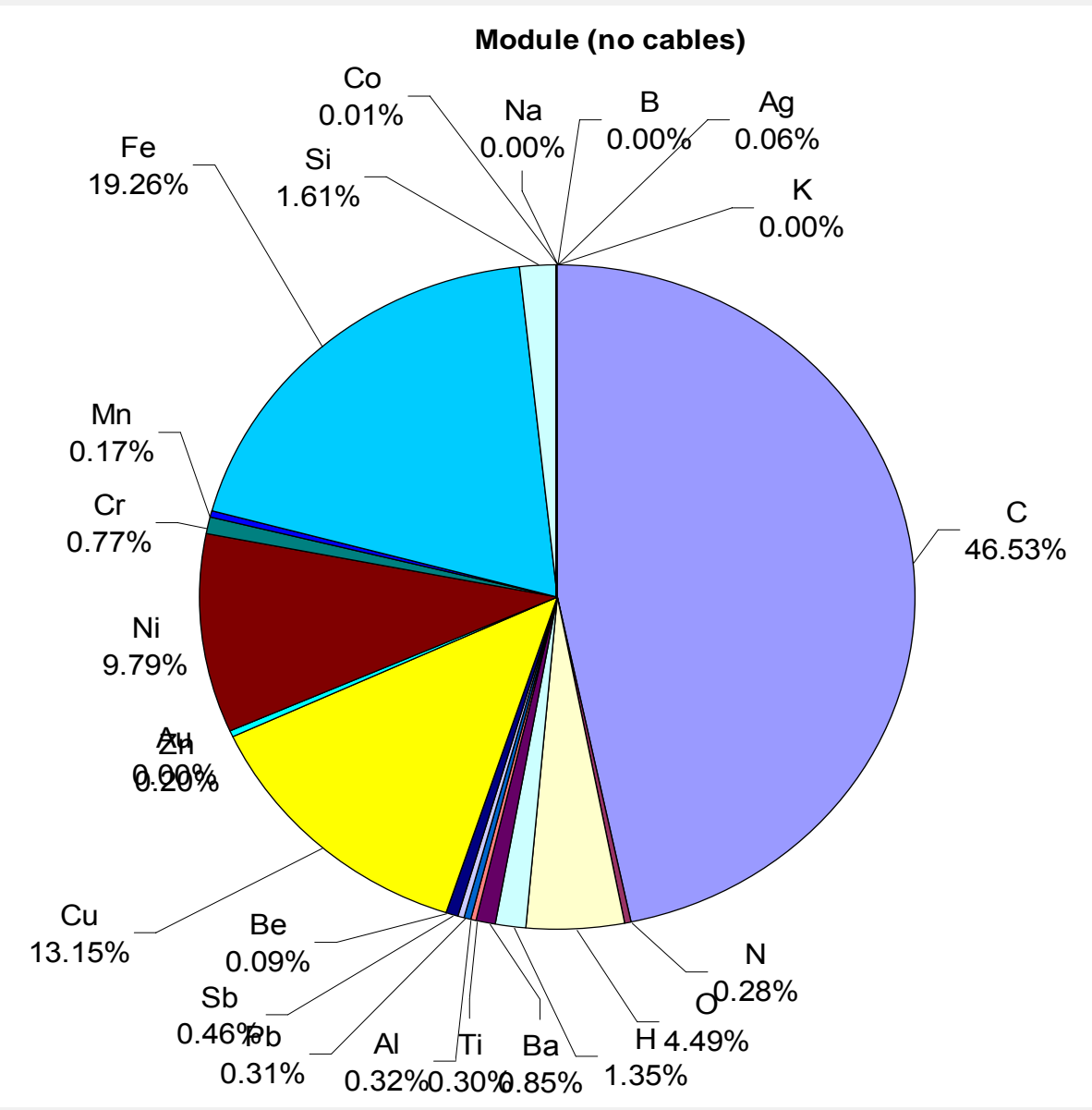




\section{Bad Channels}

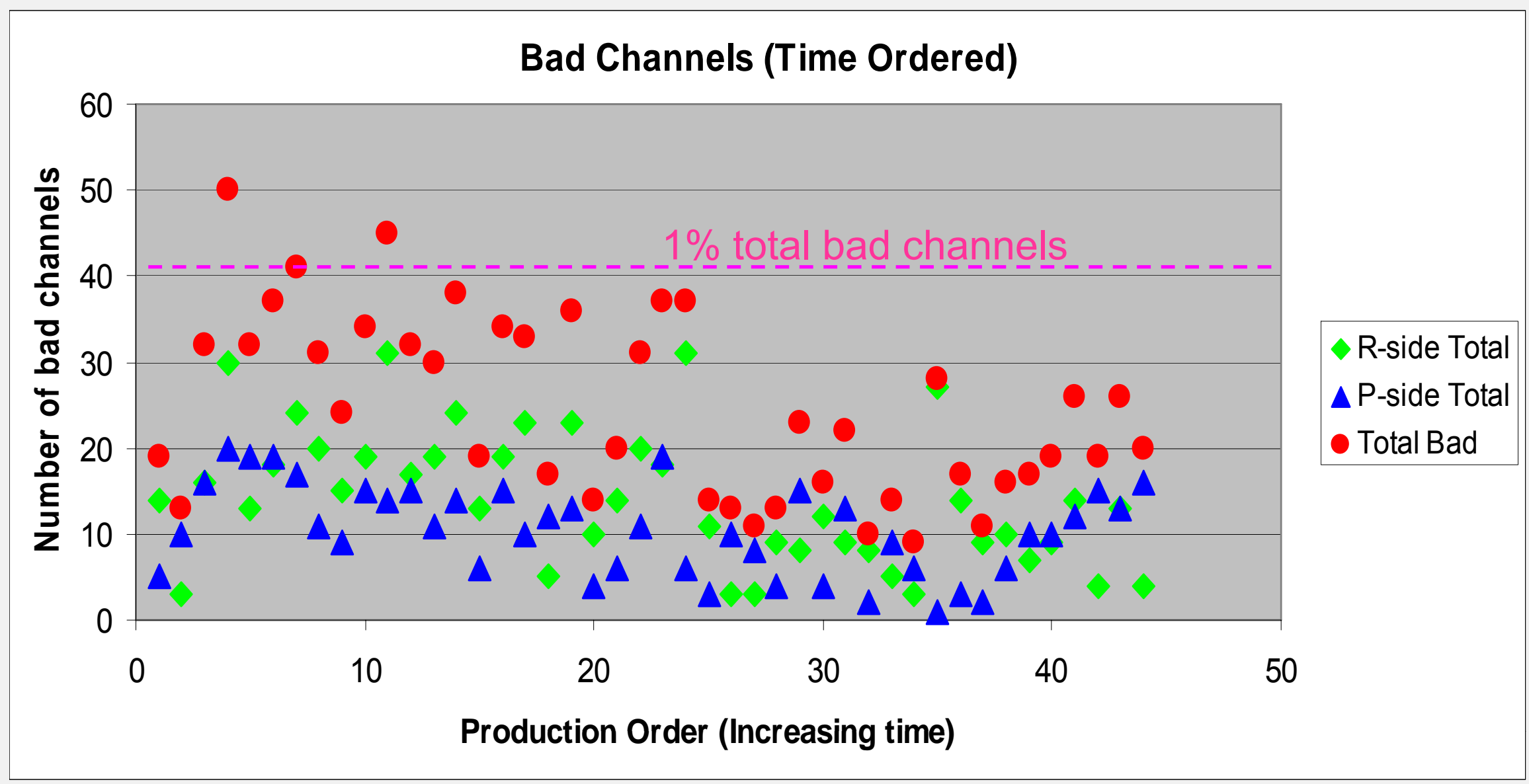

-23.5 faulty channels $(0.57 \%)$ on average

$>$ Improving throughout the production 


\section{$\mathrm{LHCb}$
THCP}

\section{LHCb VELO Collaboration}

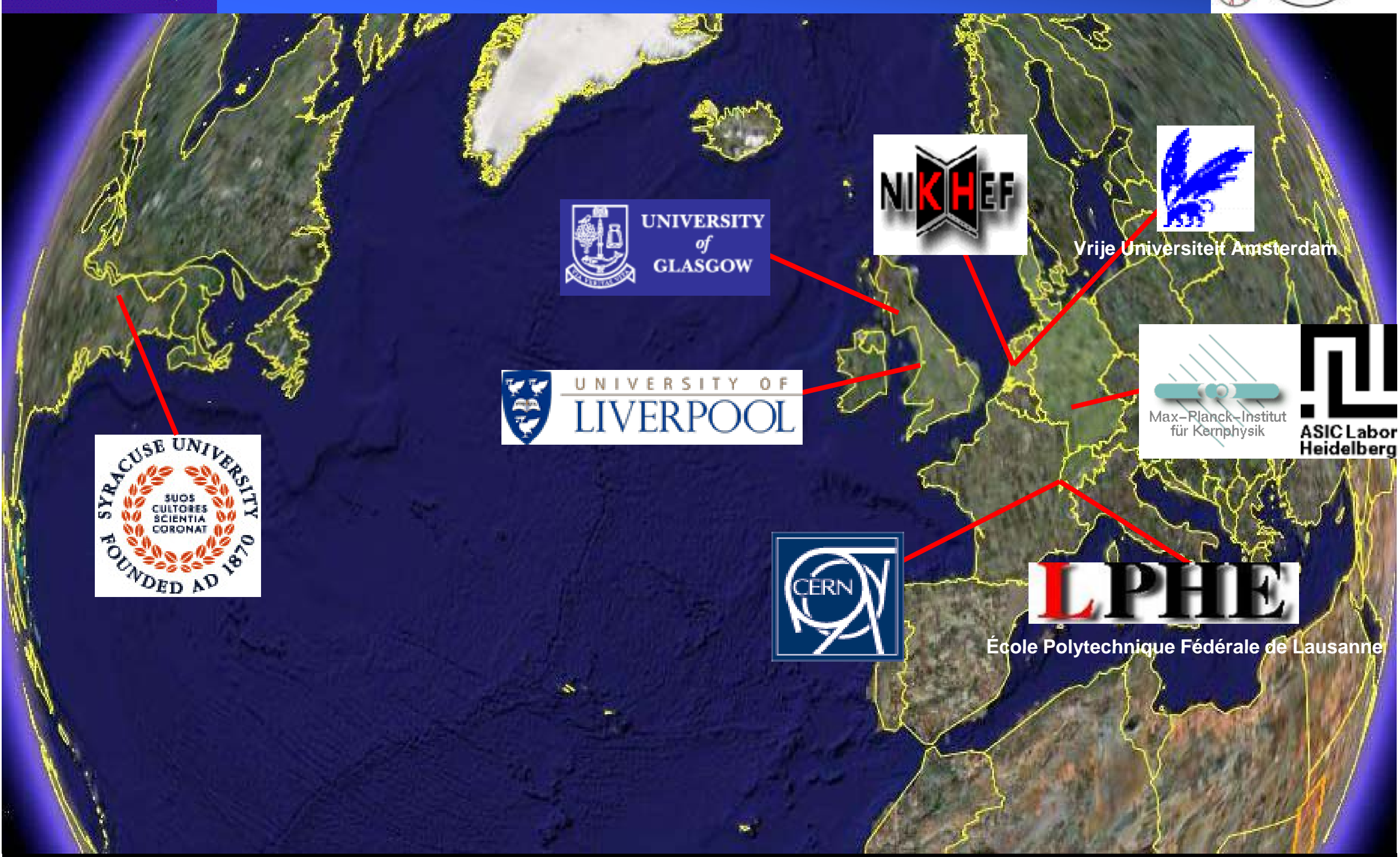




\section{Wire Bonding Quality}

-Production wire bonding performed by K\&S 8090 and 2 H\&K 710

Low re-bond rates

- Back-end: 0.6\%

- Front-end: $0.6 \%$

- Sensor-end: 0.7\%

$>$ Extremely low failure rates

- Front-end: $0.01 \%$

- Sensor-end: 0.002\%

$>$ Good pull strengths

- 8090: $10.0 \pm 1.5 \mathrm{~g}$

$-710 \mathrm{~A}: 9.4 \pm 1.5 \mathrm{~g}$

- 710B: $8.9 \pm 1.9 \mathrm{~g}$
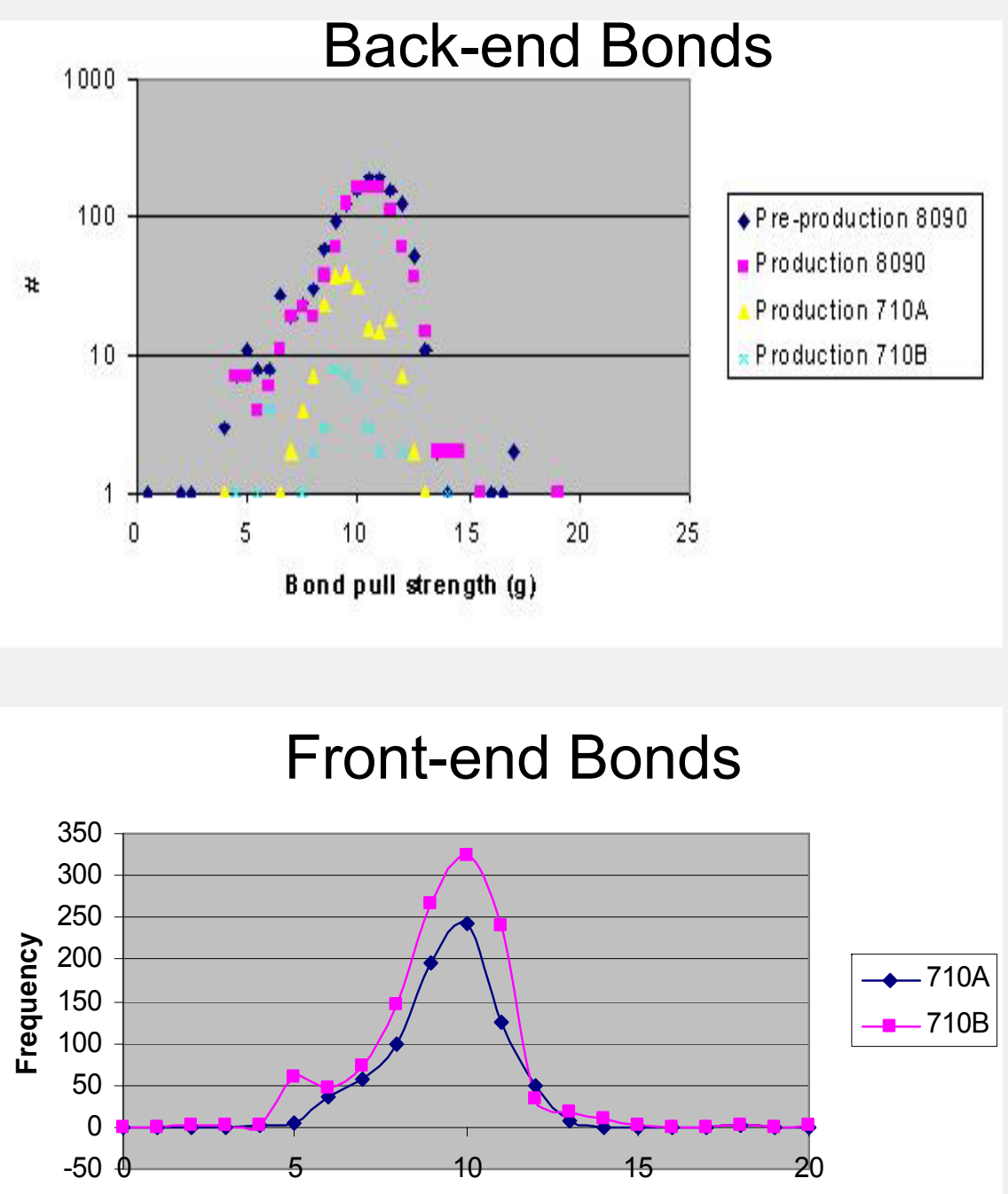

(g) 


\section{Front-end chip}

LHCb: 160x25ns deep, read out in 900 ns => SCTA not OK Design a new chip => the Beetle:

- 0.25 um CMOS ASIC

- Used in LHCb by VELO, Pile-Up system, Silicon tracker

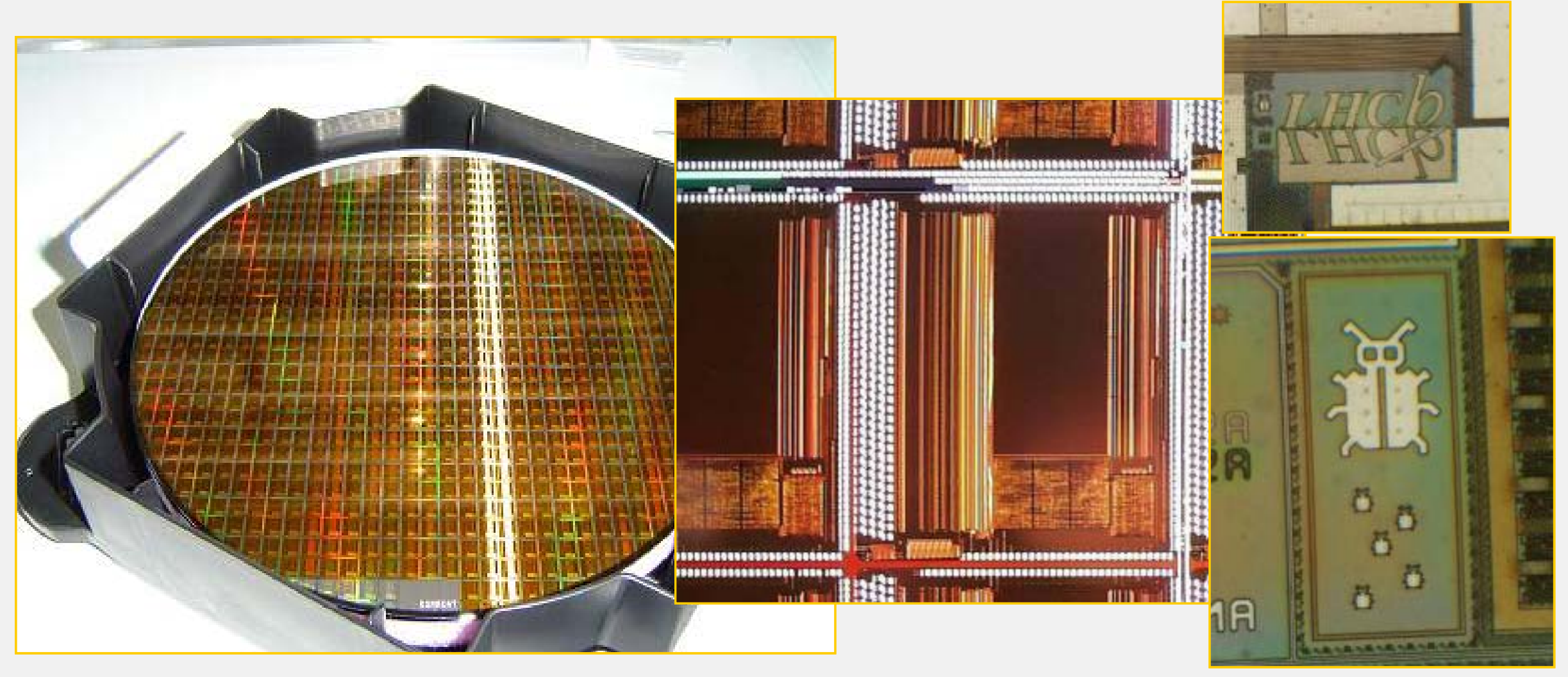

